\title{
La vie rurale en Crimée antique : Panskoe et ses environs
}

\section{Vladimir Fjodorovič Stolba}

Traducteur : Mathilde Reichler Imperiali

\section{OpenEdition \\ Journals}

Édition électronique

URL : http://journals.openedition.org/edl/365

DOI : $10.4000 /$ edl. 365

ISSN : 2296-5084

\section{Éditeur}

Université de Lausanne

\section{Édition imprimée}

Date de publication : 15 mai 2012

Pagination : 311-364

ISBN : 978-2-940331-27-7

ISSN : 0014-2026

\section{Référence électronique}

Vladimir Fjodorovič Stolba, «La vie rurale en Crimée antique : Panskoe et ses environs », Études de lettres [En ligne], 1-2 | 2012, mis en ligne le 15 mai 2015, consulté le 21 décembre 2020. URL : http:// journals.openedition.org/edl/365; DOI : https://doi.org/10.4000/edl.365 


\section{LA VIE RURALE EN CRIMÉE ANTIQUE: PANSKOE ET SES ENVIRONS}

Cet article dresse un bilan original des recherches archéologiques menées de longue date sur l'établissement de Panskoe I, l'un des monuments les plus importants et les mieux étudiés de l'époque classique tardive et du début de l'époque hellénistique dans la chôra lointaine de Chersonèse Taurique, au nord-ouest de la Crimée. Témoignant d'intéressants métissages d'éléments à la fois grecs et locaux, la culture matérielle riche et singulière de l'établissement apporte une lumière sur les aspects principaux de l'activité culturelle et domestique de ses habitants. Attendu l'histoire très courte du monument, les trouvailles archéologiques offrent, au fond, un instantané de la vie quotidienne de la paysannerie locale.

\section{Introduction}

Alors que le phénomène de la polis grecque suscite l'intérêt des scientifiques depuis plus de cent ans, les historiens sont loin d'avoir clos le débat sur la question de savoir qui des habitants de la cité résidaient à l'intérieur des murs et qui demeuraient hors les murs ${ }^{1}$. Il est toutefois évident, indépendamment de l'issue de ce débat, que la paysannerie jouait un rôle clé dans la vie de la polis, du point de vue politique, d'une part, mais aussi et surtout du point de vue militaire et économique ${ }^{2}$.

I. Voir une discussion dans M. H. Hansen, The Shotgun Method, p. 64-76, qui s'écarte du point de vue traditionnel selon lequel la paysannerie constituait à l'évidence la majorité écrasante de la population de tout Etat grec (par ex. M. Finley, "The City", p. 304 sq.; R. Osborne, Classical Landscape with Figures, p. 13; P. Cartledge, "Classical Greek agriculture», p. 132; "The economy (economies) of ancient Greece», p. 20), pour mettre son pourcentage en relation avec la taille du centre urbanistique de la polis.

2. Voir par ex. V. D. Hanson, The Other Greeks, p. 2 sq. 
Bien que l'intérêt pour les territoires ruraux, en tant que base économique des cités-Etats grecques et réelle source de la grandeur et de la prospérité de la civilisation grecque, n'ait primé que relativement tard, ce déplacement des priorités de la recherche a stimulé un développement rapide de nouvelles méthodes de prospection archéologique et d'interprétation des données, comme l'utilisation généralisée des SIG (systèmes d'information géographique) et l'expertise des sciences naturelles ${ }^{3}$. A côté des fouilles permanentes traditionnelles, cela a fourni de précieux outils aux historiens pour étudier les processus démographiques, les systèmes antiques d'établissement humain, d'aménagement foncier et d'exploitation des sols, en offrant la possibilité non seulement de poser des questions auparavant inconcevables, mais aussi d'y répondre.

La Crimée et plus particulièrement la presqu'île de Tarkhankut, située au nord-ouest, partie intégrante de la chôra lointaine de la ville antique de Chersonèse aux IV $\mathrm{IV}^{\mathrm{e}} \mathrm{II}^{\mathrm{e}}$ s. av. J.-C., représente de ce point de vue un terrain de recherche idéal. L'absence d'industrie moderne et de toute infrastructure industrielle importante a permis de préserver une intégrité presque sans égale des sites d'intérêt archéologique. Durant les prospections des années 1930-1970, de nombreux établissements ruraux ont été mis au jour sur la bande côtière de Tarkhankut, aussi bien fortifiés que non fortifiés, de tailles et de planifications diverses. Les travaux du Dzharylgach Survey Project (DSP ; 2007-2008) et du Western Crimean Archaeological Project (WCAP; 2009-2011), qui ont eu recours à des méthodes de prospection aussi bien extensives qu'intensives, ont établi que tout le territoire de la presqu'île, y compris les zones intérieures, était densément occupé au IV ${ }^{\mathrm{e}}$ siècle et au début du III $\mathrm{e}$ s. av. J.-C. ${ }^{4}$. De nombreux établissements côtiers (Masliny, Panskoe I, Bol’šoj

3. Pour un état de la question, voir A. Snodgrass, "Survey Archaeology and the Rural Landscape of the Greek City»; J. F. Cherry, «Regional Survey in the Aegean»; "Archaeology Beyond the Site»; S. E. Alcock, J. E. Gates, J. E. Rempel, "Reading the Landscape»; S. E. Alcock, J. E. Rempel, "The More Unusual Dots on the Map»; P. G. Bilde, V. F. Stolba (eds), Surveying the Greek Chora, "Introduction».

4. Pour une première approche de ces prospections, voir P. G. Bilde et al., «Džarylgač Survey Project (DSP)»; T. N. Smekalova, V. F. Stolba, Materials for the Archaeological Map of Crimea; I. N. Khrapunov, T. N. Smekalova, V. F. Stolba, «Razvedki 2009 g. v Černomorskom r-ne Avtonomnoj Respubliki Krym». WCAP fait partie intégrante du projet interdisciplinaire international Economic Models and Adaptation Strategies in a Varying Cultural and Environmental Context, dirigé par l'auteur et financé par le Danish Council for Independent Research (voir http://euxine.dk). 
Kastel', Tarpanči, Beljaus, Čajka et d'autres) ont été explorés de manière systématique et sur de longues périodes.

L'établissement Panskoe I, situé sur le littoral de la baie de Jarylgač, sur une presqu'île séparant les lacs salés Maloe Soljonoe et Džarylgač du lac Panskoe (anciennement Sasyk), est le plus éminent et incontestablement le mieux étudié des sites de la zone côtière. De 1969 à 1994, l'établissement a été exploré par la Mission archéologique de Tarkhankut de la Section de Leningrad de l'Institut d'archéologie (Académie des sciences d'URSS), devenue après 1991 l'Institut d'histoire de la culture matérielle (Académie des sciences de Russie) ${ }^{5}$. Ce site à la culture matérielle riche et originale, qui témoigne d'un enchevêtrement intéressant de composantes diverses, aussi bien grecques que locales, apporte un éclairage sur les principaux aspects de l'activité culturelle et économique de ses habitants. Compte tenu de la brièveté de l'existence de l'établissement, les trouvailles archéologiques nous offrent en effet un instantané de la vie quotidienne de la paysannerie locale.

\section{Ressources naturelles}

La partie nord-ouest de Tarkhankut, tout comme la majeure partie du territoire de la presqu'île, est une haute plaine que découpe une multitude de profonds ravins. Les sols actuels y sont constitués de tchernoziom méridional carbonaté, dont l'épaisseur peut être très variable. La couche d'humus des sols aux alentours de l'établissement de Panskoe I et autour des lacs Panskoe et Džarylgač est relativement peu profonde, mais ces sols sont dans l'ensemble fertiles et en grande partie cultivables, sauf sur les coteaux de l'élévation de Tarkhankut. Il n'est guère étonnant que dans de nombreux cas les établissements antiques de Tarkhankut se trouvent plutôt sur des terrains aux sols fertiles. Toutefois, à la lumière de nouvelles découvertes du projet WCAP, la thèse de A. N. Ščeglov, qui

5. A. N. Ščeglov, Severo-Zapadnyj Krym v antičnuju èpokhu, p. 46-49 et 80-82; "25 let rabot Tarkhankutskoj èkspedicii»; "Un établissement rural en Crimée», p. 239-257; A. Chtcheglov, Polis et chora; V. F. Stolba, «Dom IV v. do n.è. na poselenii Panskoe I». Sur l'histoire de l'étude archéologique de l'établissement, voir L. Hannestad, V. F. Stolba, A. N. Ščeglov (eds), Panskoye I, vol. 1, p. 23 sq. et 32 sq. Une bibliographie détaillée consacrée à l'établissement de Panskoe I est disponible sur internet à l'adresse http://euxine.dk/?page_id=516. 
présumait l'absence d'établissement sur les terrains totalement impropres à la culture des sols ${ }^{6}$, doit être revue.

Une particularité caractéristique de cette région de la Crimée est l'absence totale de rivières et de lacs d'eau douce. Compte tenu du taux élevé d'ensoleillement, des faibles précipitations et d'une évaporation presque deux fois plus importante que les précipitations, l'approvisionnement en eau était et reste un problème vital pour les habitants de la région. Les eaux souterraines, souvent puisées à une profondeur importante, sont la principale source d'eau potable. On trouve des puits en grande quantité dans les embouchures des grands ravins, où la nappe phréatique est la plus proche. Les ressources en eaux étant plutôt maigres, la proximité d'une source d'eau potable devait à coup sûr être un critère décisif dans la formation du système antique d'établissement humain dans cette région.

Les recherches géomorphologiques montrent que la ligne du littoral a connu des modifications conséquentes au cours des 2500 dernières années. La découverte des restes d'une propriété grecque du IV $\mathrm{s}$. av. J.-C., engloutie par les eaux et enfouie dans la vase du lac Panskoe, laisse penser que le niveau de la mer devait être à cette époque de quelques mètres plus bas que le niveau actuel ${ }^{7}$. Les résultats conjoints de recherches sur les dépôts de fonds du lac salé de Džarylgač, menées en 2005 par une expédition de l'Université d'Aarhus et par l'Institut de limnologie (Académie des sciences de Russie), ont considérablement enrichi nos connaissances de la géomorphologie de cette zone ${ }^{8}$. Comme l'ont montré ces travaux, au moment de l'apparition d'établissements ruraux grecs sur ce territoire, ce lac d'origine lagunaire était déjà totalement isolé de la mer par un cordon littoral de sable et s'était transformé en un

6. A. N. Ščeglov, Severo-Zapadnyj Krym v antičnuju èpokhu, p. 24.

7. N. S. Blagovolin, A. N. Ščeglov, "Archaeological, Paleogeographic, and Geomorphological Researches in the Lake Sasyk (Panskoye) Region ", p. 291-294 et 298.

8. D. A. Subetto, T. V. Sapelko, V. F. Stolba, «Issledovanija paleolimnologov v Krymu»; D. A. Subetto et al., "Environmental and Black Sea Level Changes in the Holocene as recorded in Lakes Saki and Dzharylgach, Crimean Peninsula»; I. Ju. Neustrueva et al., «Rekonstrukcija paleobiogeografičeskikh i paleoekologičeskikh uslovij oz. Džarylgač (Severo-Zapadnyj Krym) v pozdnem golocene po paleontologičeskim dannym». 
plan d'eau peu profond et fortement minéralisé, impropre à la navigation et à la pêche.

Les modifications qui ont affecté la flore de la presqu'île sont dues en grande partie à l'impact anthropique, en plus des changements induits par les variations climatiques. Le paysage actuel de l'ouest de la Crimée se caractérise par une végétation steppique et semi-désertique à prédominance de graminées, d'herbes et d'arbustes xérophiles à longue période de végétation, ainsi que par une absence totale de forêts et de taillis. Durant la seconde moitié du IV s. av. J.-C., période de l'expansion maximale de la chôra de Chersonèse, la végétation de Tarkhankut jouissait sans doute d'une plus grande diversité et était apparemment de type forêt-steppe ${ }^{9}$, type qui s'est manifestement maintenu dans la zone littorale de la presqu'île jusqu'à la période romaine ${ }^{10}$.

\section{Vestiges archéologiques aux alentours de Panskoe I}

Deux établissements antiques ont été mis au jour à proximité immédiate de Panskoe I, appelés Panskoe II et Panskoe III et situés respectivement sur les côtes sud-est et nord du lac du même nom (fig. 1). Des explorations du lac Panskoe ont permis de découvrir encore les vestiges d'une autre propriété du IV e s. av. J.-C. engloutie et enfouie dans la vase du lac (Panskoe IV) ${ }^{11}$. En 1991, à $400 \mathrm{~m}$ au sud-est de l'établissement de Panskoe I, l'auteur de cet article identifia, dans un champ de labour, les vestiges d'une petite propriété qui fut nommée Panskoe V.

Les fouilles de prospection à l'extrémité nord du cordon littoral de Džarylgač, sur le territoire du bourg de Mežvodnoe, ont permis d'y révéler encore la présence d'une autre riche propriété grecque, datée en

9. A. N. Ščeglov, Severo-Zapadnyj Krym v antičnuju èpokhu, p. 24 sq.; L. Hannestad, V. F. Stolba, A. N. Ščeglov (eds), Panskoye I, vol. 1, p. 21 ; A. N. Ščeglov et al., "Zemledelie na poselenii Panskoe I (Severo-Zapadnyj Krym) v IV - načale III v. do n.è.», p. 65 ; Z. V. Januševič, A. N. Ščeglov, «Palaeoethnobotanical Material», p. 329 sq.

IO. A. N. Ščeglov, Severo-Zapadnyj Krym v antičnuju èpokhu, p. 25 sq.; P. D. Podgorodeckij, Severo-Zapadnyj Krym, p. 33 sq.

II. N. S. Blagovolin, A. N. Ščeglov, "Archaeological, Paleogeographic, and Geomorphological Researches in the Lake Sasyk (Panskoye) Region », p. 293 sq. et 298. 
Fig. 1 - Monuments archéologiques des environs de l'établissement de Panskoe I.

1. Jarylgač-Nord, 2. Jarylgač-Est, 3. Panskoe V, 4. Jarylgač 2 (S11-022).

dernier examen des $\mathrm{IV}^{\mathrm{e}}-\mathrm{III}{ }^{\mathrm{e}}$ s. av. J.-C. ${ }^{12}$. La vie s'y poursuivit toutefois par intermittence jusqu'au milieu du $\mathrm{II}^{\mathrm{e}} \mathrm{s}$. av. J.-C., ainsi qu'en témoignent des fragments de céramique à vernis noir découverts par hasard au cours de travaux de construction sur le territoire du bourg et que j'ai pu examiner en $2010^{13}$.

L'origine de l'occupation par l'homme de la baie de Jarylgač et de la région du lac Džarylgač remonte toutefois au Néolithique. On trouve des

I2. T. N. Vysotskaja, «Drevnegrečeskoe poselenie v pos. Mežvodnoe».

I3. J'exprime ma reconnaissance à Azime Kemalova, directrice du musée archéologique à Černomorskoe, qui m’a aimablement fait connaître ces trouvailles. 
traces d'un établissement de cette période (Jarylgač-Severnoe) au nordest de Panskoe I, dans une brèche de la falaise littorale ${ }^{14}$. L'établissement de relativement grande envergure de Jarylgač-Vostočnoe, situé à $800 \mathrm{~m}$ au sud-est de Panskoe I, présente des matériaux de l'époque du Bronze tardif et du haut Moyen Age ${ }^{15}$. C'est peut-être à la phase primitive de cet établissement qu'il faut rapporter la sépulture centrale du kourgane K1, explorée en 1994 sur le territoire de la nécropole antique de Panskoe I ${ }^{16}$. Des traces de vie sédentaire de l'époque du Bronze tardif (seconde moitié du deuxième millénaire avant notre ère) ont également été relevées dans d'autres lieux : au sud et au nord du lac Džarylgač, en amont du ravin de Kirleut, au nord-est du village de Vodopojnoe, ainsi qu'au sud du bourg de Zajcevo. L'exploration géomagnétique et les sondages archéologiques de quelques-uns de ces sites ont révélé la présence de constructions d'habitation rectangulaires en pierre couplées à des constructions de forme circulaire qui servaient manifestement d'enclos pour le bétail ${ }^{17}$. Outre sur l'établissement Jarylgač-Vostočnoe mentionné plus haut, des vestiges du Moyen Age ont également été découverts à la périphérie orientale de Panskoe I, où fut mise au jour en 1989 une sépulture à catacombe médiévale de l'époque de Saltovo. C'est à cette même période (VII ${ }^{\mathrm{e}}$ IX ${ }^{\mathrm{e}}$ siècles) qu'appartient le nouvel établissement médiéval Jarylgač 2 (S10-022), découvert par l'auteur de cet article au cours de fouilles menées en 2010 sur la rive occidentale de la baie de Jarylgač (fig. 1).

\section{Superficie et structure de l'établissement}

A la différence de la plupart des établissements ruraux de cette région, constitués de propriétés indépendantes, fortifiées ou non, le site qui nous occupe est original en ce qu'il se présente comme une agglomération

I4. N. S. Blagovolin, A. N. Ščeglov, "Archaeological, Paleogeographic, and Geomorphological Researches in the Lake Sasyk (Panskoye) Region", p. 287, pl. 180.

I5. Ibid., p. 288 sq., pl. 181.

I6. A. N. Ščeglov, L. Hannestad, V. F. Stolba, "Raboty rossijsko-datskoj Tarkhankutskoj èkspedicii v Krymu», p. 337 ; A. N. Ščeglov, "Issledovanija Tarkhankutskoj èkspedicii", p. 275; V. F. Stolba, E. Rogov, Panskoye I, vol. 2, p. 66, n. 11.

17. P. G. Bilde et al., "Džarylgač Survey Project (DSP)»; T. N. Smekalova, V. F. Stolba, Materials for the Archaeological Map of Crimea, p. 30 sq., fig. 9-10. 
à la fois de propriétés collectives indépendantes et de structures bâties d'habitation de plus grande taille (fig. 2-3.1). Près d'une dizaine de tels complexes bâtis sont susceptibles d'être préalablement identifiés sur le terrain. Ils sont disposés de manière plutôt compacte, situés les uns des autres à une distance qui va de quelques mètres à quelques dizaines de mètres, et seules deux constructions (U13 et U14) se trouvent à un certain éloignement du noyau principal, en raison du relief du terrain. La superficie totale de l'établissement s'élève à un petit peu plus de quatre hectares. Trois imposants dépôts de cendres, dont l'un, à la périphérie ouest du secteur central U7, a été intégralement fouillé en 1985, apparurent à la suite du déblaiement de l'établissement après un incendie et des destructions qui le touchèrent au troisième quart du $\mathrm{IV}^{\mathrm{e}} \mathrm{s}$. av. J.-C. Une nécropole de kourganes, rattachée à l'établissement, est située $150 \mathrm{~m}$ plus au nord et compte une soixantaine de tertres, ainsi qu'une quantité importante d'inhumations en pleine terre comprises dans l'espace séparant les kourganes les uns des autres ${ }^{18}$.

\section{Stratigraphie et chronologie}

Apparu au dernier quart du $\mathrm{V}^{\mathrm{e}}$ s. av. J.-C. comme avant-poste fortifié de la polis d'Olbia, Panskoe I passe vers 360 av. J.-C. sous la dépendance de Chersonèse, avec laquelle elle conserve d'étroits liens jusqu'à la ruine catastrophique de l'établissement vers 270 av. J.-C. C'est à cette même période que remonte l'abandon des exploitations rurales sur tout le territoire de l'Etat de Chersonèse, tout comme dans d'autres centres grecs du nord de la mer Noire. Il y a des raisons de croire que ce ne sont pas seulement les incursions de nomades, mais aussi plusieurs autres facteurs, les modifications de l'environnement n'étant pas le moindre d'entre eux, qui ont pu être à l'origine des transformations soudaines et de grande ampleur survenues dans la région du Pont septentrional. On trouve une confirmation de cela dans le fait que la désagrégation effective des établissements ruraux et la contraction de la chôra des villes grecques furent précédées d'une crise monétaire, manifestement même d'une crise

I8. V. F. Stolba, E. Rogov, Panskoye I, vol. 2, p. 9. 
Fig. 2 - Etablissement et nécropole de Panskoe I.

Plan schématique des secteurs fouillés.

économique globale, dont on trouve des traces dans tous les plus grands centres de la région ${ }^{19}$.

Il se dégage de la série des couches anthropiques deux horizons stratigraphiques principaux, qui correspondent aux étapes signalées plus haut de la vie de l'établissement. Pour l'essentiel, seule la stratification

19. V. F. Stolba, "Monetary Crises in the Early Hellenistic Poleis of Olbia, Chersonesos and Pantikapaion"; "The Oath of Chersonesos and the Chersonesean Economy in the Early Hellenistic Period". 
Fig. 3.1 - Maquette des complexes U7 et U6 dans la partie centrale de l'établissement (reconstitution de A. N. Ščeglov, maquette de I. O. Zavadskaja).

supérieure (l'horizon A) a fait l'objet de recherches archéologiques systématiques; c'est à celle-ci que se rapportent des complexes intégralement fouillés comme U16, U13 et U14, ainsi que l'établissement Panskoe III. C'est également à cette même période, si l'on en juge par le matériel récolté, qu'il faut faire remonter l'établissement de Panskoe II situé non loin. Les couches inférieures de l'horizon B, dont les plus anciennes (horizon B2) se rapportent à l'époque où existait la forteresse d'Olbia, restent encore insuffisamment étudiées et ne sont actuellement répertoriés que dans le secteur U7, dans la partie centrale du site ${ }^{20}$, ainsi que sous les fondations des bâtiments de l'époque chersonésite du complexe U2.

Bien que des restes de constructions de périodes plus tardives n'y aient pas encore été révélés, des trouvailles isolées de fragments de coupes mégariennes laissent penser que pour une courte période, au dernier tiers du III s. av. J.-C., la vie reprit cours à Panskoe I, comme dans plusieurs autres établissements ruraux de Crimée occidentale ${ }^{21}$.

20. A. N. Ščeglov, "Un établissement rural en Crimée», p. 242 sq. et 261, fig. 7-8; V. F. Stolba, «Dom IV v. do n.è. na poselenii Panskoe I».

2I. L. Hannestad, V. F. Stolba, H. Blinkenberg Hastrup, «Black-Glazed, Red-Figure and Grey Ware Pottery», p. 129 sq.; L. Hannestad, "The Dating of the Monumental Building U6 at Panskoye I», p. 191. 


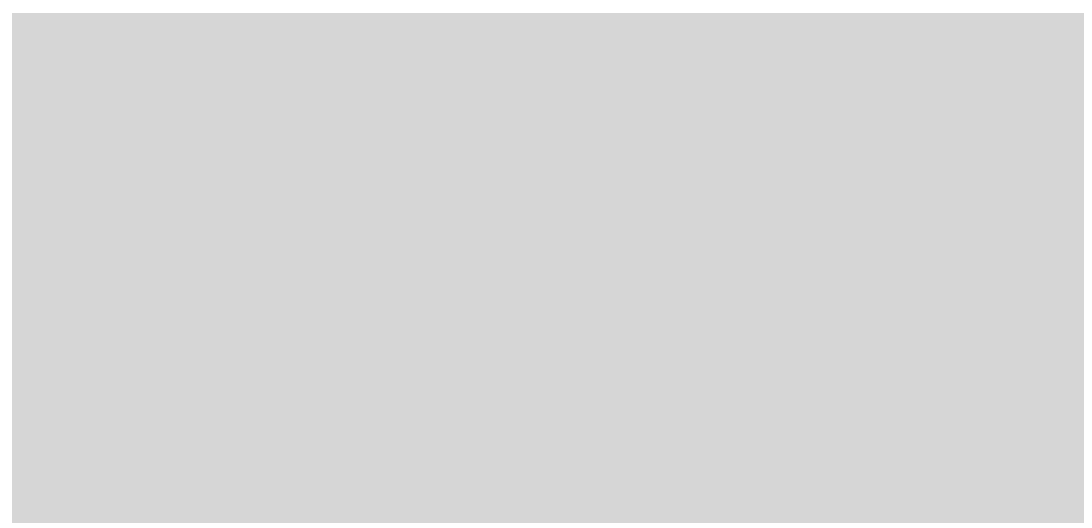

Fig. 3.2 - Vue aérienne du bâtiment monumental U6.

\section{Les complexes explorés de l'établissement}

Plusieurs complexes bâtis ont été intégralement explorés au cours de plus d'un quart de siècle de travaux archéologiques sur l'établissement. Au nombre de ceux-ci se trouve le bâtiment monumental U6 (fig. 3.2) qui a fait l'objet d'une publication particulière ${ }^{22}$. Son origine et son activité sont entièrement liées à l'étape chersonésite de la vie de l'établissement. De dimensions de 34,5 x 34,5 m et d'une surface totale de près de $1190 \mathrm{~m}^{2}$, ce bâtiment de deux étages à plan carré était constitué de plus de trois dizaines de pièces, agencées en une ou deux rangées autour d'une vaste cour intérieure. Au centre de la cour se trouvait un puits taillé dans la roche. Les pièces habitables se situaient de toute évidence dans la partie sud-ouest du bâtiment, d'où provient l'essentiel des graffiti contenant des marques de propriétaires ${ }^{23}$. La pièce 3 , située le long du mur nord-ouest du bâtiment, remplissait une fonction particulière. Il y fut trouvé 37 amphores, dont 30 de production chersonésite, qui étaient apparemment entreposées au premier étage. Les inscriptions trouvées sur les amphores contiennent les noms d'au moins trois propriétaires différents, ce qui conduit à considérer ce local comme un lieu de stockage collectif $^{24}$. Plusieurs autres pièces du bâtiment étaient peut-être à usage

22. L. Hannestad, V. F. Stolba, A. N. Ščeglov, Panskoye I, vol. 1.

23. V. F. Stolba, "Graffiti and Dipinti", p. 230 sq.

24. Ibid., p. 231. 


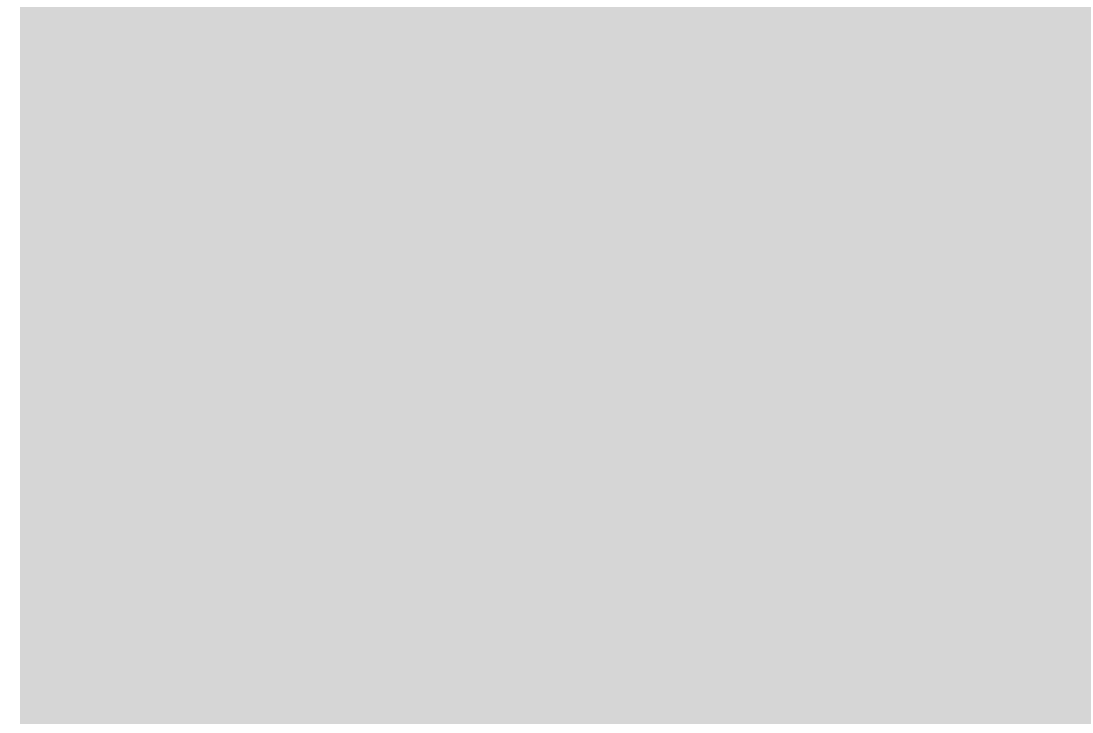

Fig. 4 - Secteur central U7. Vue de la tour nord (II) du fort olbien ancien.

collectif. Ainsi, le graffito $\delta \alpha \mu o ́ \sigma($ ov) sur le fond d'un plat à vernis noir, trouvé dans la cour près de la pièce $5^{25}$, montre qu'à cet endroit (également au premier étage) pouvait se trouver une pièce consacrée aux repas en commun.

Le secteur central U7, adjacent au bâtiment U6 du côté nord-est, est le plus ancien noyau de l'établissement, avec des vestiges bien conservés du fort quadrangulaire de la période d'Olbia, de 42,5 x 42,5 m et ceint à ses angles de quatre tours rondes (fig. 4) ${ }^{26}$. Avec l'arrivée de colons de Chersonèse, la fortification perd son rôle défensif et son territoire accueille de nouveaux bâtiments. Débordant la ligne des enceintes défensives, les constructions de l'époque chersonésite se développent vers le sud-ouest, en direction du lac, et finissent par pratiquement doubler

25. Ibid., p. 234, H 32, pl. 4; p. 152 et 157.

26. La récente affirmation d'A. V. Bujskikh (Prostranstvennoe razvitie Khersonesa Tavričeskogo $v$ antičnuju èpokhu, p. 151 sq.), selon laquelle le complexe U7, avec les tours rondes et les constructions habitables, est un ensemble homogène, appartenant à une seule époque de construction et qui est lié exclusivement à la présence chersonésite dans cette région, rend perplexe et n'a rien à voir avec l'état réel des choses. Son opinion ne se fonde malheureusement que sur des plans schématiques et incomplets et non sur une analyse stratigraphique ni sur une prise de connaissance personnelle du site. 
Fig. 5 - Plan du secteur central U7.

la surface de la forteresse olbienne primitive. Groupés autour de cours intérieures collectives, les bâtiments sont accolés les uns aux autres et se présentent comme seize ensembles distincts de maisons d'habitation (fig. 5). Si les couches de l'horizon stratigraphique supérieur (A) sont intégralement explorées dans le secteur U7, les couches plus anciennes de l'établissement ne sont encore connues qu'insuffisamment. Les couches de l'horizon stratigraphique B1 ont été dégagées notamment lors de l'exploration du dépôt de cendre qui recouvrait la tour III. A cet endroit ont été révélés les vestiges d'un bâtiment adjacent à la tour et au mur d'enceinte et ayant existé approximativement entre 360 et 340 av. J.-C. Ce complexe extra muros représente actuellement le plus ancien témoignage 
Fig. 6 - A gauche, plan des bâtiments U13 et U13A; à droite, plan du bâtiment U7-15.

de la présence chersonésite tant sur l'établissement de Panskoe que, plus largement, dans toute la Crimée nord-occidentale ${ }^{27}$.

$\mathrm{Au}$ nombre des complexes étudiés en totalité mais dont les résultats des recherches n'ont pas encore été publiés, il y a également, comprises dans une couche unique, deux propriétés chersonésites partiellement détruites par les eaux du lac, U13 (fig. 6) et U14, situées respectivement à $100 \mathrm{~m}$ et $70 \mathrm{~m}$ au nord-est du complexe U7. De nombreux complexes de l'horizon supérieur (U1-3, U9, U10) demeurent partiellement explorés ou complètement inexplorés scientifiquement. Parmi eux, le secteur U2, étudié entre 1987 et 1994, mérite une attention particulière: à cet endroit de la périphérie nord-est de l'établissement a été découvert un bloc de maisons de même type, d'une surface standard d'environ $260 \mathrm{~m}^{2}$, assemblées en un tout ${ }^{28}$. Des maisons de planification similaire, mais de surface sensiblement moindre (d'environ $195 \mathrm{~m}^{2}$ ), ont également été découvertes dans le secteur U7 (bâtiment 15) (fig. 6).

27. V. F. Stolba, «Dom IV v. do n.è. na poselenii Panskoe I».

28. A. N. Ščeglov, L. Hannestad, V. F. Stolba, «Raskopki poselenija Panskoe I v Krymu»; "Raboty rossijsko-datskoj Tarkhankutskoj èkspedicii v Krymu»; A. N. Ščeglov et al., «Rossijsko-datskie raskopki na poselenii i nekropole Panskoe I». 


\section{La nécropole}

La surface totale de la nécropole s'élève à un peu moins de quatre hectares. De 1969 jusqu'à nos jours, plus d'un tiers de son territoire a été exploré, qui compte trente-trois kourganes et une soixantaine d'inhumations en pleine terre comprises dans les espaces entre les kourganes ${ }^{29}$. L'immense majorité des complexes funéraires découverts se rapporte à la première moitié et au troisième quart du $\mathrm{IV}^{\mathrm{e}} \mathrm{s}$. av. J.-C. Des inhumations plus tardives de la période chersonésite ont été beaucoup moins bien étudiées; il s'en trouve encore peut-être dans la partie non fouillée du cimetière.

Le rite funéraire dont atteste la nécropole présente une étonnante combinaison d'éléments grecs et barbares (scythes et taures). Les inhumations se faisaient dans des fosses rectangulaires, des tombes à niche, dont on trouve de très proches analogues dans les sites funéraires du Boug et du Dniestr inférieurs, mais elles se faisaient aussi dans des caveaux de brique crue ou dans des fosses garnies de brique crue et couvertes d'un remblai de terre. Ce remblai était affermi à sa base à l'aide d'une structure circulaire en pierre appelée crépis. On plaçait fréquemment, du côté sud-ouest de la crépis, un autel de sacrifice en pierre creusé d'un renfoncement en forme de coupe et d'une rainure pour l'écoulement, identique aux autels de sacrifice de la nécropole d'Olbia ${ }^{30}$. Les inhumations étaient souvent accompagnées de banquets funéraires. Fréquentes sont les trouvailles (souvent in situ) de monuments tombaux anthropomorphiques en calcaire, de type dit chersonésite (fig. 7.3-4). Une des tombes (K36 M3) se distingue par la présence d'une sculpture primitive anthropomorphique au faciès barbare (fig. 7.5).

On trouve en général dans les tombes un à deux squelettes, et jusqu'à quatre inhumations successives dans quelques cas. Les défunts étaient enterrés sur le dos en position allongée, parfois sur le côté, les jambes légèrement repliées. Ce genre de squelettes recroquevillés, que l'on associe pour cette période à la population non grecque, représentée par la culture archéologique de Kizil-Koba ou par les Taures historiques, a été relevé dans quinze cas. Presque tous les squelettes dont le sexe a pu être identifié appartenaient à des femmes. Curieusement, dans plusieurs

29. Voir pour plus de détails V. F. Stolba, E. Rogov, Panskoye I, vol. 2.

30. A. N. Ščeglov, "Un établissement rural en Crimée», fig. 14.3-4. 
Fig. 7 - Sculpture taillée dans le calcaire provenant de l'établissement et de la nécropole: 1. figurine d'un héros local (?) (photo de Niels Hannestad), 2. figurine féminine de culte (photo de Niels Hannestad), 3-5. stèles funéraires anthropomorphes. 
cas, les tombes qui ont servi à plusieurs inhumations successives contenaient la combinaison d'un squelette d'homme étendu et d'un squelette de femme recroquevillé. Compte tenu du fait que les squelettes recroquevillés ne sont pas, loin de là, l'unique confirmation de la présence non grecque dans l'établissement, il est tout à fait naturel de considérer de telles inhumations communes comme le témoignage de mariages mixtes qui avaient cours au sein de la population ${ }^{31}$.

L'inventaire des objets funéraires dans les tombes est assez standard. Il comporte de la vaisselle pour le vin ou l'eau (amphores et récipients à boire) et pour les parfums, des couteaux, des bijoux, etc. Dans les tombes masculines, on trouve parfois des armes (flèches, lances), plus rarement des strigiles. Le matériel issu des tombes féminines est composé de miroirs, aiguilles, fusaïoles et autres objets du quotidien de la femme. Il convient peut-être d'examiner à part les inhumations en fosse dans le sol, dont l'inventaire est très pauvre, voire complètement inexistant.

Durant les années de fouilles de la nécropole, il a été trouvé plusieurs squelettes présentant les marques évidentes d'une mort violente. Si l'on considère la stratigraphie du complexe U7, qui présente des couches d'incendie clairement distinctes, cela laisse penser que la vie de l'établissement, même avant l'époque de sa fin tragique durant le premier tiers du III ${ }^{\mathrm{e}}$ s. av. J.-C., est loin d'avoir toujours été paisible.

\section{Economie, production, artisanat}

C'est sans le moindre doute possible la culture des céréales et l'élevage qui ont constitué, tout au long de l'histoire de l'établissement, la base de l'activité économique de ses habitants. La pêche devait être développée dans la zone côtière. La chasse, si elle a été pratiquée, ne jouait probablement qu'un rôle accessoire.

Les photographies prises de l'espace et les clichés aériens d'archives montrent qu'aux alentours de Panskoe, tout comme sur la presqu'île d'Héraclée, les terres étaient aménagées en un système normalisé de découpage en parcelles rectangulaires. De tels lots, d'une surface de 20-21 ha (682 $\pm 2 \mathrm{~m}$ sur 315-320 m) et aux angles orientés vers les points

3I. V. F. Stolba, «Multicultural Encounters in the Greek Countryside», p. 329-334. 
cardinaux, ont été notamment identifiés au sud du lac Panskoe (fig. 1) ${ }^{32}$. Des fragments d'un cadastre antique ont également été découverts plus à l'ouest, aux environs du site de Černomorskoe (Ak-Mečet) ${ }^{33}$, de même que dans la partie centrale de la presqu'île de Tarkhankut, entre les villages de Karadža (l'actuel Olenevka) et Kunan (l'actuel Krasnosel'skoe), où Ščeglov est parvenu à mettre en évidence d'après les clichés photographiques un vaste système de bornage orthogonal ${ }^{34}$. Dans certains cas isolés, on peut même identifier sur le terrain de telles traces de bornage. Leur état de conservation est nettement moins bon que sur la presqu'île d'Héraclée, à l'exception d'un lot près du cap Ojrat ${ }^{35}$. En raison des spécificités environnementales locales, les murets de bornage étaient souvent construits non pas en pierre, mais en terre, ce qui leur laissait peu de chances de survie, compte tenu des conditions actuelles de labour en profondeur. L'un de ces fragments d'ancien cadastre, constitué d'un système de petits talus de terre délimitant des parcelles d'environ 80 x $90 \mathrm{~m}$, a néanmoins été découvert et mesuré en 2007, au cours du projet DSP dans un champ de labour au nord du lac Džarylgač (DSP07-F08). Les trouvailles concomitantes de céramique modelée rendent très probable leur appartenance à la haute époque hellénistique.

Les principales cultures agricoles pratiquées au $\mathrm{IV}^{\mathrm{e}}$ siècle et au début du III s. av. J.-C. dans les champs des alentours de la baie de Jarylgač, tout comme dans le reste du territoire de Tarkhankut, étaient les graminées. On a mis en évidence la présence de pollens de cultures céréalières dans les sédiments de fond du lac Džarylgač, tant pour la couche de la période subboréale que pour la couche de la période subatlantique ${ }^{36}$. Des trouvailles de graines de céréales permettent de juger plus en détail de la composition des semences. Nombre d'entre elles,

32. A. N. Ščeglov et al., "Zemledelie na poselenii Panskoe I», p. 53 sq., fig. 2.

33. A. N. Ščeglov, "Issledovanie sel'skoj okrugi Kalos Limena», p. 240, fig. 2; Severo-Zapadnyj Krym v antičnuju èpokhu, p. 87, fig. 43; "Utilisation de la photographie aérienne dans l'étude du cadastre de Chersonésos Taurique», p. 62 et 69, fig. 8-9; A. Chtcheglov, Polis et chora, p. 119. En dépit de la tradition, qui reconnait dans ce site la Kalos Limèn des auteurs anciens (Arrien, Périple du Pont-Euxin, 19.5-20.1) et des inscriptions de Chersonèse (IOSPE I $\left.\mathrm{I}^{2}, 352.21 ; 353 ; 401.8,21\right)$, cette identification est fragile.

34. A. N. Ščeglov, «Utilisation de la photographie aérienne dans l'étude du cadastre de Chersonésos Taurique», p. 62 sq. et 72, fig. 14.

35. A. N. Ščeglov, «Zemel'nyj nadel u mysa Ojrat».

36. T. V. Sapelko, D. A. Subetto, V. F. Stolba, «Vlijanie rel'efa na razvitie rastitel'nogo pokrova Kryma v golocene», p. 331. 
identifiées dans plusieurs complexes de l'établissement de Panskoe I et datant d'une période entre le début du IV ${ }^{\mathrm{e}}$ siècle et le début du III ${ }^{\mathrm{e}} \mathrm{s}$. av. J.-C., ont été examinées en détail par A. N. Ščeglov, N. N. Kuz’minova, Z. V. Januševič et E. S. Čavčavadze ${ }^{37}$, ainsi que par G. A. Paškevič ${ }^{38}$. Des matériaux complémentaires nous sont parvenus en 2004 à l'occasion du déblaiement d'un silo à grain partiellement démonté par des pillards dans le complexe U10 (horizon stratigraphique A) ${ }^{39}$. Outre les caryopses de blé tendre hérisson (Triticum aestivo-compactum), qui constituaient la base des exportations de céréales chersonésites, on y trouve également de l'orge. Les décomptes quantitatifs indiquent qu'elle occupait souvent la deuxième place et qu'elle pouvait parfois prévaloir dans les semences, comme c'est le cas dans les trouvailles de Kerkinitis ${ }^{40}$.

Des modifications notables touchent l'assortiment des céréales cultivées au début du III ${ }^{\mathrm{e}}$ s. av. J.-C.: le seigle se fait de plus en plus fréquent dans les trouvailles de cette époque, même si on en rencontre déjà quelques graines dans des complexes du troisième quart du $\mathrm{IV}^{\mathrm{e}} \mathrm{s}$. av. J.-C. (Panskoe I/U2, tranchée 2/fosse 2). Ces trouvailles comptent au nombre des plus anciens témoignages de l'adoption du seigle en culture de champs non seulement pour la région de la mer Noire, mais aussi au-delà. Sur le territoire de Tarkhankut, en dehors de Panskoe, des caryopses de seigle ont aussi été révélés dans des trouvailles du début du III ${ }^{\mathrm{e}}$ s. av. J.-C. dans l'établissement de Masliny, dans une propriété sur la baie Vetrenaja, à Beljaus ${ }^{41}$, ainsi que dans l'établissement Kel'šejkh 1, dans la partie centrale de la presque-île, lors de fouilles effectuées en $2010^{42}$. Dans deux échantillons du bâtiment U6 à Panskoe, le seigle était la principale composante, avec $73,2 \%$ et $81,9 \%$, alors que les

37. A. N. Ščeglov et al., "Zemledelie na poselenii Panskoe I"

38. G. A. Paškevič, «Archaeobotanical studies on the northern coast of the Black Sea», p. 524, fig. 7.

39. Non publiés. Ont été découverts du blé tendre, de l'orge et du seigle (identification de l'auteur). Leurs proportions relatives n'ont pas été comptées.

40. G. A. Paškevič, Paleoetnobotaničeskie nakhodki na territorii Ukrainy, p. 15 sq.; V. A. Kutajsov, «Agrarnaja istorija Kerkinitidy (I)», p. 83.

4I. O. D. Daševskaja, "Antičnaja bašnja na gorodišče Beljaus», p. 89; Z. V. Januševič, Kul'turnye rastenija Jugo-Zapada SSSR po paleobotaničeskim issledovanijam, p. 135-138; Kul'turnye rastenija Severnogo Pričernomor' ja, p. 54; A. N. Ščeglov, Severo-Zapadnyj Krym v antičnuju èpokhu, p. 104.

42. Fouilles de l'auteur; matériau non publié. 
$26,8 \%$ et $18,1 \%$ restants étaient constitués de blé ${ }^{43}$. Le pourcentage de seigle est encore plus élevé dans deux échantillons de Masliny, avec $90 \%$ et $95 \% 44$.

Ces deux dernières trouvailles de Panskoe et de Masliny sont particulièrement remarquables, car le seigle n'ayant jamais été destiné à l'exportation, à la différence du blé, sa culture ne laissait pas espérer de réels revenus. Si l'on en croit les jugements des auteurs anciens, ses qualités gustatives ne pouvaient pas non plus faire concurrence au blé. Pline l'Ancien le qualifie de nourriture exécrable, désagréable à l'estomac et que l'on ne consomme que pour éviter la faim (NH 18.141: deterrimum et tantum ad arcendam famem). La question qui se pose donc naturellement est celle des raisons d'une adoption si précoce du seigle comme culture unique et de sa popularité inattendue auprès de la paysannerie locale.

Les modifications dont il est question surviennent au moment où, si l'on en juge par les données dont nous disposons, éparses mais très révélatrices dans leur ensemble, Chersonèse commence à s'enfoncer dans une longue et profonde crise économique. Comme j'ai tenté de le démontrer ailleurs, cette crise a touché non seulement la Crimée occidentale, mais pratiquement tous les centres grecs et le monde barbare du nord de la mer Noire, et elle a apparemment été la conséquence de brusques changements climatiques et environnementaux ${ }^{45}$. Il est évident

43. Z. V. Januševič, Kul'turnye rastenija Jugo-Zapada SSSR po paleobotaničeskim issledovanijam, p. 134-136; A. N. Ščeglov, Severo-Zapadnyj Krym v antičnuju èpokhu, p. 104 sq.; Z. V. Januševič, A. N. Ščeglov, «Palaeoethnobotanical Material», p. 327-329.

44. Z. V. Januševič, Kul'turnye rastenija Jugo-Zapada SSSR po paleobotaničeskim issledovanijam, p. 134 et 137; K. Wasylikowa et al., "East-Central Europe», p. 232 sq.

45. V. F. Stolba, "Monetary Crises in the Early Hellenistic Poleis of Olbia, Chersonesos and Pantikapaion"; "The Oath of Chersonesos and the Chersonesean Economy in the Early Hellenistic Period». Outre dans le serment de Chersonèse (IOSPE $\left.\mathrm{I}^{2}, 401\right)$, on trouve des échos de cette crise dans le fameux décret en l'honneur de Protogène (IOSPE I², $32 \mathrm{~A}, 25,59 ; \mathrm{B}, 81$ ), qui signale une famine ( $\sigma \imath \tau o \delta \varepsilon i ́ \alpha)$ et de mauvaises récoltes (óopí $\alpha$ ). Dans le but de vérifier cette hypothèse et d'obtenir des données paléo-environnementales complémentaires, l’auteur a initié en 2005 le projet international interdisciplinaire "Northern Black Sea Area in the 1st Millennium BC: Long-Term Human History and Climate Changes». Voir I. Ju. Neustrueva et al., «Rekonstrukcija paleobiogeografičeskikh i paleoekologičeskikh uslovij oz. Džarylgač (Severo-Zapadnyj Krym) v pozdnem golocene po paleontologičeskim dannym»; D. A. Subetto, T. V. Sapelko, V. F. Stolba, «Issledovanija paleolimnologov v Krymu»; D. A. Subetto et al., "Environmental and Black Sea Level Changes in the Holocene as 
que ces changements, qui ont entraîné des conséquences catastrophiques pour l'économie des cités-Etats grecques, ne pouvaient pas ne pas laisser leur empreinte sur le caractère de l'agriculture locale. Miser sur une culture plus résistante a pu être une mesure efficace d'adaptation, dans le contexte d'une crise s'aggravant et de conditions climatiques défavorables, ce qui a été et qui reste toujours une stratégie traditionnelle de la paysannerie pour réduire le risque de mauvaise récolte, parallèlement à la diversification des semences et au semis dans les interlignes ${ }^{46}$. Laugmentation dans les semences du pourcentage de seigle, moins exigeant envers la qualité du sol et plus résistant à la rigueur des hivers et à la sécheresse des étés, répondait on ne peut mieux à cette nécessité. Bien que quelques trouvailles de graines de Secale cereale aient été faites dans des complexes de Myrmèkion et Hermonassa remontant déjà au $\mathrm{VI}^{\mathrm{e}}$ s. av. J.-C. ${ }^{47}$, c'est manifestement aux tribus scythes de la zone steppique et de la forêt-steppe que la population de la périphérie agraire de Chersonèse doit d'avoir assimilé cette culture. C'est de là précisément, si l'on en croit les données publiées ${ }^{48}$, que proviennent les trouvailles les plus anciennes de seigle pur. Il convient de signaler que des trouvailles de graines isolées sont connues dans cette région dès l'époque du Bronze tardif 49 .

Parmi les autres cultures agricoles attestées à Panskoe par des trouvailles paléobotaniques, il faut signaler la lentille erviliaire (Vicia

recorded in Lakes Saki and Dzharylgach, Crimean Peninsula»; I. Ju. Neustrueva et al., "Rekonstrukcija paleobiogeografičeskikh i paleoekologičeskikh uslovij oz. Džarylgač (Severo-Zapadnyj Krym) v pozdnem golocene po paleontologičeskim dannym "; T. V. Sapelko, D. A. Subetto, V. F. Stolba, «Vlijanie rel'efa na razvitie rastitel'nogo pokrova Kryma v golocene».

46. J. S. Wood, C. G. Knight, "Cross cultural perspectives on human adjustments to arid margins", p. 90, 92, 96 sq.; R. Longhurst, "Household Food Strategies in Response to Seasonality and Famine», p. 27-29 et 33; Th. W. Gallant, Risk and Survival in Ancient Greece, p. 36-41. Comparer avec D. Rahmato, Famine and Survival Strategies, p. 195 sq.

47. I. B. Zeest, "Arkhaičeskie sloi Germonassy», p. 54; I. T. Kruglikova, Sel'skoe khozjajstvo Bospora, p. 182; G. A. Paškevič, "Archaeobotanical studies on the northern coast of the Black Sea", p. 522 sq.

48. K. Wasylikowa et al., «East-Central Europe», p. 231 ; G. A. Paškevič, «New evidence for plant exploitation by the Scythian tribes during the Early Iron Age in the Ukraine", p. 597-599.

49. G. A. Paškevič, «Early Farming in the Ukraine», p. 267 sq.; «Paleoetnobotanični doslidžennja Skhidnoj Ukraïny", p. 13. 
ervilia) et la lentille cultivée (Lens culinaris), ainsi que le millet (Panicum miliaceum $)^{50}$. La résistance notoire du millet à la sécheresse et le fait qu'il soit peu exigeant envers la qualité des sols, de même que sa courte période d'arrivée à maturité (60 à 110 jours) ${ }^{51}$, qui permet de faire jusqu'à deux récoltes par an, sont de ces qualités importantes qui ont conféré à cette culture, de même qu'au seigle, une importance stratégique en condition de famine et de mauvaises récoltes. Bien que des trouvailles de millet soient attestées dans des sites antiques du littoral nord de la mer Noire, particulièrement à Olbia et dans les établissements de son district agraire ${ }^{52}$, cette culture n'avait pas acquis auprès des Grecs la popularité dont elle jouissait chez les tribus de la zone steppique et de la forêt-steppe ${ }^{53}$. Il est remarquable que le millet ait continué dans des périodes ultérieures à jouer un rôle important dans l'économie locale des habitants des steppes de Russie et d'Ukraine méridionale, où jusqu'au $\mathrm{XIX}^{\mathrm{e}}$ siècle il faisait partie intégrante de la ration céréalière des Tatares de Crimée, et en représentait même souvent la partie principale.

Parmi les cultures pratiquées dans les environs de Panskoe, il faut enfin mentionner la vigne, révélée dans l'établissement par des trouvailles aussi bien de pépins que de fragments de ceps ${ }^{54}$. Le plus ancien

50. A. N. Ščeglov, Severo-Zapadnyj Krym v antičnuju èpokhu, p. 106 sq.; A. N. Ščeglov et al., "Zemledelie na poselenii Panskoe I (Severo-Zapadnyj Krym) v IV - načale III v. do n.è. », p. 57 et 63; Z. V. Januševič, Kul'turnye rastenija Severnogo Pričernomor'ja, p. 54; Z. V. Januševič, A. N. Ščeglov, "Palaeoethnobotanical Material", p. 329.

51. E.g. Ph. M. Smith, «Minor Crops», p. 308 sq.

52. G. A. Paškevič, "Archaeobotanical studies on the northern coast of the Black Sea», p. 515-523. Ces données sont en accord avec l'indication d'Hérodote (IV.17.1) selon laquelle le millet appartient au nombre des cultures pratiquées par des tribus des Callipides et des Alazones, à la périphérie nord de la polis d'Olbia.

53. Z. V. Januševič, «Die Kulturpflanzen Skythiens», p. 87 sq. et 92; G. A. Paškevič, «New evidence for plant exploitation by the Scythian tribes during the Early Iron Age in the Ukraine"; "Archaeobotanical studies on the northern coast of the Black Sea", p. 526 sq. Les plus anciennes trouvailles de millet sur ce territoire remontent à l'époque du chalcolithique (culture de Trypillja et étape tardive de la culture DnieprDonets). Voir A. V. Nikolova, G. A. Paškevič, «K voprosu ob urovne razvitija zemledelija Tripol'skoj kul'tury», p. 89; G. A. Paškevič, "Paleoethnobotanični doslidžennja Skhidnoj Ukraïny", p. 12.

54. A. N. Ščeglov et al., "Zemledelie na poselenii Panskoe I (Severo-Zapadnyj Krym) v IV - načale III v. do n.è.», p. 57 et 63; Z. V. Januševič, A. N. Ščeglov, «Palaeoethnobotanical Material», p. 329. 
de ces témoignages se rapporte au début du $\mathrm{IV}^{\mathrm{e}}$ s. av. J.-C., ce qui permet d'avancer que la vigne a été cultivée tout au long de l'histoire de l'établissement. La présence parmi les trouvailles du complexe U6 d'un fouloir de pierre ${ }^{55}$, ainsi que d'un couteau de jardin, qui pouvait servir aussi bien à la taille de branches qu'à celle des ceps, pourrait être une indication indirecte de l'existence de la viticulture, et peut-être de l'horticulture. Toutefois, à la différence de ce qu'on observe dans les environs immédiats de Chersonèse, sur la presqu'île d'Héraclée et sur la presqu'île Majačnyj, où la vigne était la culture principale avec un taux d'occupation des terres cultivées parcellées allant jusqu'à $60 \%-70 \%{ }^{56}$, elle ne pouvait pas à cet endroit, à la périphérie nord du territoire agraire de la polis, opposer une réelle concurrence aux céréales. Le littoral sud de Tarkhankut, où on a exploré, près du cap Ojrat, un lot de terre ayant accueilli un vignoble parfaitement conservé de type chersonésite, qui se présente comme des rangées parallèles de murs de pierre ${ }^{57}$, constitue de ce point de vue une exception, de même que les alentours d'Eupatoria, où l'on trouve également des témoignages révélateurs de la spécialisation dans la viniculture de commerce ${ }^{58}$.

L'inventaire des outils agricoles est complété par de rares trouvailles de socs en métal destinés à optimiser l'efficacité de l'araire ${ }^{59}$. Bien que l'usage de socs métalliques soit connu dans l'Antiquité dès l'époque homérique (Hom. Il. 23.238 sqq.), la plupart des trouvailles de tels objets dans le territoire du nord de la mer Noire date de l'époque romaine ${ }^{60}$. Seuls deux socs, provenant du comblement du complexe 17 du site de la ville d'Elizavetovskoe, dans le delta du Don, sont proches, par leur datation, des objets de Panskoe ${ }^{61}$. Le principal outil de moisson était la

55. L. Hannestad, V. F. Stolba, A. N. Ščeglov, Panskoye I, vol. 1, p. 62, pl. 33.1-3.

56. Voir S. F. Strželeckij, Klery Khersonesa Tavričeskogo, p. 149.

57. A. N. Ščeglov, «Zemel'nyj nadel u mysa Ojrat».

58. I. V. Jacenko, «Vinodel'nja na poselenii Čajka»; A. B. Kolesnikov, I. V. Jacenko, "Le territoire agricole de Chersonèse taurique dans la région de Kerkinitis», p. 311-317.

59. A. N. Ščeglov, Severo-Zapadnyj Krym v antičnuju èpokhu, p. 107, fig. 57; E. Ya. Rogov, "Metal Objects», p. 262, n ${ }^{\circ} \mathrm{K} 147$, pl. 168. Un autre soc a encore été découvert en 1983 dans le bâtiment U7/pièce 51.

6o. Voir I. T. Kruglikova, Sel'skoe khozjastvo Bospora, p. 164-167, avec la bibliographie.

6I. K. K. Marčenko, V. G. Žitnikov, V. P. Kopylov, Die Siedlung Elizavetovka am Don, p. 174, Abb. 72.1 . 
faucille en fer, dont plusieurs exemplaires ont été trouvés à Panskoe ${ }^{62}$. Le chaume restant après moisson était manifestement labouré avec la terre, ou brûlé sur pied, ce qui se pratique encore de nos jours dans cette région.

Le soin du stockage de la récolte était manifestement l'affaire de chaque ménage ou collectif familial. Nous n'avons à l'heure actuelle pas connaissance de dépôt qui aurait subvenu aux besoins de toute la population. Le mode le plus courant de stockage de la récolte était de le conserver dans des silos à grain ordinaires, creusés dans le sol et qui pouvaient se trouver aussi bien à l'intérieur des bâtiments qu'à l'extérieur. La conservation des céréales dans de telles fosses, connues chez les Grecs sous le nom de oipós (Démosthène 8.45), était largement pratiquée dans le monde antique. Des expériences modernes ont démontré la grande efficacité de ces greniers enterrés ${ }^{63}$. A Panskoe, une concentration de sept silos de la seconde moitié du IVe s. av. J.-C. a été découverte à la périphérie orientale de l'établissement, sous les vestiges du complexe du bâtiments U2. D'une profondeur de plus d'un mètre et d'un diamètre au niveau du fond atteignant $1,70 \mathrm{~m}$, ils se présentent sous les deux formes principales qu'on leur connaît par ailleurs: en cloche ou en poire. En comparaison, on n'a trouvé dans l'établissement que relativement peu de pithoi. L'éloignement des grands centres de production de céramique et la difficulté de transporter des conteneurs de grande capacité n'expliquent pas cette préférence, car des trouvailles de pithoi volumineux ne sont pas rares, par exemple, dans l'établissement de Beljaus et dans l'exploitation rurale de la baie Vetrenaja, aux environs de Černomorskoe ${ }^{64}$. Il est naturel de penser que ce sont plutôt ici des considérations d'économie qui ont pesé dans ce choix, ce que semble également indiquer la pratique largement répandue dans l'établissement de réutilisation sur de longues périodes des amphores, y compris pour le stockage du grain. Dans l'une de ces amphores, trouvée dans la pièce 3 du complexe U6, on a découvert un entassement important de pas moins de 30000 à

62. A. N. Ščeglov, Severo-Zapadnyj Krym v antičnuju èpokhu, p. 107, fig. 57 sq.; E. Ya. Rogov, «Metal Objects», p. 262, nos K 141-147, pl. 168; V. F. Stolba, E. Rogov, Panskoye I, vol. 2, ${ }^{\circ} \mathrm{K} 68$, pl. 75.

63. J. D. Currid, A. Navon, «Iron Age Pits and the Lahav (Tell Halif) Grain Storage Project».

64. A. Chtcheglov, Polis et chora, p. 124 sq.; P. D. Diatroptov, «Pifosy s gorodišča Beljaus s graffiti». 
50000 grains carbonisés ${ }^{65}$. Les pieds de ces amphores étaient en général brisés à dessein, afin de pouvoir les ficher plus commodément dans le sol.

Comme il en a été fait mention plus haut, l'élevage était l'autre grande branche de l'économie paysanne de la chôra de Chersonèse ${ }^{66}$. A en juger par les restes d'ossements en provenance du bâtiment U6 ${ }^{67}$, du complexe central U7, ainsi que de la nécropole, le petit bétail à cornes (chèvres et moutons) constituait la base du complexe faunistique de l'établissement. Ainsi, dans l'une des inhumations de la nécropole (K43 M3), datée du troisième quart du IV ${ }^{\mathrm{e}} \mathrm{s}$. av. J.-C., on a trouvé un ensemble de 334 astragales de mouton ou de chèvre ${ }^{68}$. Sept autres astragales semblables viennent de la tombe M037. Enfin, dans la pièce 3 du bâtiment U6, on a découvert une coupe contenant 134 astragales de mouton ou de chèvre ${ }^{69}$. Tous ces ossements devaient provenir d'au moins 119 animaux. Parmi le matériel faunistique du complexe U7, A. K. Kasparov a attribué à des moutons et à des chèvres 284 os qui provenaient de 17 individus ${ }^{70}$. Il convient toutefois de noter que ces trouvailles proviennent de contextes chronologiquement distincts. Quoi qu'il en soit, on peut cependant penser que le cheptel de moutons était bien plus important que ce qu'indiquent les chiffres minimaux dont nous disposons. Durant la période antique, la prédominance de petit bétail dans le cheptel était caractéristique pour beaucoup de sites barbares de la zone steppique, de même que pour les centres grecs du nord de la mer Noire ${ }^{71}$. Aux époques ultérieures également, et ce jusqu'à nos jours, l'élevage ovin

65. Z. V. Januševič, A. N. Ščeglov, «Palaeoethnobotanical Material», p. 327.

66. Cf. la discussion dans S. J. Hodkinson, "Animal Husbandry in the Greek Polis» sur le rôle de l'élevage dans l'économie de la polis.

67. A. K. Kasparov, "Osseous Remains».

68. V. F. Stolba, E. Rogov, Panskoye I, vol. 2, p. 107 et 360 sq.

69. A. N. Ščeglov, «Monumental Building U6», p. 41, pl. 19.3; non pris en compte dans le panorama de A. K. Kasparov, «Osseous Remains».

70. Matériaux non publiés.

7I. P. D. Liberov, "K istorii skotovodstva i okhoty na territorii Severnogo Pričernomor'ja v èpokhu rannego železa", p. 120; O. P. Žuravl'jov, Istorija fauny i tvarynnyctva Nyžn'ogo Pobužžja v antičnyj čas, p. 16-18. L'établissement d'Elizavetovka (K. K. Marčenko, V. G. Žitnikov, V. P. Kopylov, Die Siedlung Elizavetovka am Don, p. 168 sq.) constitue une exception à cette règle, de même que certains sites de la région du Dniepr et du Kuban (V. I. Calkin, «Domašnie i dikie životnye Severnogo Pričernomor'ja v èpokhu rannego železa», p. 90 ; N. V. Anfimov, "Skotovodstvo u sindo-meotskikh plemjon Prikuban'ja», p. 5), où prédominent les bovins. 
reste une composante importante de l'économie de Tarkhankut. Les voyageurs des XVIII e et XIX ${ }^{\mathrm{e}}$ siècles louaient unanimement l'étonnante qualité de la laine des moutons locaux, qui n'avait pas son pareil sur tout le territoire de la Crimée.

Les porcins, les chevaux et le gros bétail avaient également une place importante au sein du cheptel de l'établissement de Panskoe. Le parcage des moutons se faisait de toute évidence sur les versants à pente douce de l'élévation de Tarkhankut, sur lesquels le labour était impraticable, comme cela se fait encore de nos jours. Le parcage des animaux sur les terres en jachère n'est pas non plus à exclure. Cette pratique avait cours dans certains lieux de Grèce (Théocrite Idyll. 35.99), bien que les accords officiels d'affermage l'interdisaient en général (IG XII 7.62 et XIV 645) ${ }^{72}$. D'après des bergers de la région, ce mode de parcage des ovins sur les terres en jachère se pratique souvent en Crimée occidentale encore actuellement.

Bien que toutes ces données concernant la faune soient tout à fait significatives, la question du lieu dans lequel étaient tenus tous ces animaux reste actuellement ouverte. Compte tenu de la densité de construction que l'on observe à Panskoe, de la taille et du caractère de l'agencement des bâtiments individuels, on ne peut pas imaginer que le bétail ait pu se tenir sur le territoire même de l'établissement. Apparemment, de manière analogue à ce qu'on peut observer pour les sites de l'époque du Bronze de la région ${ }^{73}$, les abris et les enclos étaient situés à une certaine distance des maisons d'habitation, à la périphérie de l'établissement, encore insuffisamment explorée à l'heure actuelle.

Les espèces auxquelles appartiennent les ossements aviaires du complexe U7 n'ont malheureusement pas pu être identifiées; ceux du bâtiment U6 proviennent quant à eux exclusivement d'animaux sauvages. Les trouvailles de coquilles d'œufs sur les lieux des banquets funéraires laissent toutefois supposer l'existence également d'une pratique de l'élevage avicole. Autant qu'on puisse en juger par les rares ossements d'animaux sauvages retrouvés (lièvre, renard, gerboise sauteuse

72. H. Michell, The Economics of Ancient Greece, p. 54. Sur l'élevage ovin chez les Grecs anciens, voir O. Brendel, Die Schafzucht im alten Griechenland. Voir également S. Isager, J. E. Skydsgaard, Ancient Greek Agriculture, p. 91-93, qui s'appuient sur les témoignages d'Aristote.

73. P. G. Bilde et al., "Džarylgač Survey Project (DSP)», p. 109-112, fig. 1; T. N. Smekalova, V. F. Stolba, Materials for the Archaeological Map of Crimea, p. 30, fig. 9. 
[Allactaga jaculus Pall.]), la chasse ne jouait pas de rôle décisif dans l'économie de l'établissement. Les produits de la mer devaient enrichir de manière plus significative l'alimentation de la population locale, compte tenu de la position littorale de l'établissement et des trouvailles d'arêtes de poisson ou de restes de crabes, de nombreuses coquilles de moules et d'huîtres, ainsi que de vestiges d'équipement de pêche (aiguilles en os pour la confection des filets; lests de pierre ou de céramique pour les filets, hameçons de bronze, pointes de harpons en bronze ou en fer).

Les données dont on dispose sont insuffisantes pour décrire de manière exhaustive les autres domaines de l'activité économique et domestique des habitants de Panskoe, comme le travail des métaux, le filage, le tissage ou la taille de la pierre, même si toutes ont à l'évidence existé. Les fouilles de l'établissement, et en particulier de la nécropole, ont donné une collection impressionnante d'objets de bronze, de fer, de plomb, ainsi que d'argent et d'or ${ }^{74}$. Il convient en particulier de mentionner un groupe de six objets de fer trouvés dans le bâtiment U6 et interprétés comme étant des outils de charpenterie ${ }^{75}$. Des trouvailles éparses de moules en pierre témoignent également de l'existence sur le site d'une production de fonderie.

Quelques exemples de sculptures en calcaire assez primitives (un relief d'Héraclès et de petits autels portatifs en forme d'oiseaux), trouvés dans le bâtiment U6, sont à ranger selon Ščeglov sur la liste des objets de production locale ${ }^{76}$. On peut ajouter à leur nombre des luminaires taillés dans le calcaire, dont un est à l'évidence une copie d'un objet semblable en céramique, ainsi que la figurine en calcaire d'un héros local (?), le bras droit replié, une coupe à la main et qui prend appui de la main gauche sur un bouclier circulaire, et la figurine cultuelle d'une femme de type barbare (fig. 7.1-2). Tous les monuments funéraires anthropomorphiques de calcaire découverts sur le territoire de la nécropole étaient eux aussi produits dans l'établissement, selon toute vraisemblance. Ces monuments funéraires, qui se présentent sous différentes formes, sont d'une

74. E. Ya. Rogov, «Metal Objects»; V. F. Stolba, E. Rogov, Panskoye I, vol. 2, p. 326340 , ${ }^{\text {os }} \mathrm{K} 1-92$, pl. 68-76.

75. E. Ja. Rogov, «Nabor stoljarno-plotnič ikh instrumentov iz raskopok zdanija U6 poselenija Panskoe I»; E. Ya. Rogov, «Metal Objects», p. 264, F 164-169, pl. 166, 169, 170.

76. A. N. Ščeglov, "Cult sculpture, Altars, Sacred Vessels and Votivs», p. 213 sq. et 221, ${ }^{\text {os }}$ G 1, G 5-6, pl. 143, 145, 146. 
qualité de fabrication très variable, allant des objets les plus finement sculptés aux objets très primitifs et grossièrement façonnés (fig. 7.3-4). Cette diversité témoigne selon moi de l'absence sur place d'une production spécialisée de ces sculptures. Chaque collectivité familiale abordait apparemment ce problème au gré des nécessités, à la mesure de ses forces et selon les capacités artisanales et artistiques de ses représentants.

L'importation d'objets de céramique en provenance de Chersonèse et d'Olbia, ainsi que d'autres centres étrangers, ne satisfaisait pas complètement les besoins en céramique de cuisine et de table de la paysannerie locale. Une part importante du complexe céramique des établissements ruraux de la chôra de Chersonèse est constituée de céramique modelée, dont l'essentiel était à l'évidence fabriqué sur place (fig. 8). Des traces d'une telle production ont été révélées à Panskoe dans l'une des pièces de la partie nord du bâtiment U6 ${ }^{77}$. Même si les récipients sont dans leur immense majorité modelés à la main, certains d'entre eux portent des traces d'utilisation, lors de la finition, d'un tour de potier rudimentaire $^{78}$. Ces objets étaient vraisemblablement cuits dans des fosses ou des fourneaux primitifs situés quelque part à l'extérieur de l'établissement, car il n'a pas été donné de les découvrir, ni par des moyens archéologiques, ni lors des recherches géophysiques.

A Panskoe, comme dans n'importe quel autre site, l'inventaire des types de céramique modelée évolue au $\mathrm{IV}^{\mathrm{e}}$ et au début du III $\mathrm{e}$ s. av. J.-C. Pour l'essentiel, les types modelés tombent en désuétude à la fin de la période en question, supplantés surtout par des ouvrages de poterie. Le pourcentage de céramique modelée reste cependant élevé dans l'assortiment de l'établissement et dépasse considérablement les chiffres correspondants de plusieurs autres établissements ruraux antiques du Boug inférieur (tableau 1) ${ }^{79}$. Il ne fait pas de doute que durant la dernière période de l'existence de Panskoe, la céramique modelée et la céramique tournée constituent un ensemble cohérent. Si pour l'essentiel la céramique tournée d'importation est représentée dans les complexes tardifs par des formes de table, la céramique modelée, dans la vie quotidienne,

77. V. F. Stolba, «Handmade Pottery», p. 180, pl. 134.a.

78. Ibid., p. 182, pl. 135.

79. On trouve un pourcentage encore plus élevé de céramique modelée dans les établissements ruraux des régions reculées de Tarkhankut, comme dans l'établissement de Kel'šejkh 1, que nous avons fouillé en 2010-2011. 
Fig. 8 - Trouvailles céramiques de l'établissement et de la nécropole. 


\begin{tabular}{|c|c|c|c|}
\hline \multirow{2}{*}{$\begin{array}{l}\text { blocs (maisons } \\
\text { d'habitation) }\end{array}$} & \multicolumn{3}{|c|}{$\%$ de l'assortiment céramique } \\
\hline & objets entiers & fonds & $\begin{array}{l}\text { quantité totale de } \\
\text { fragments }\end{array}$ \\
\hline 1 & 15,4 & 25,0 & 50,2 \\
\hline $2-3$ & 28,6 & 30,8 & 55,5 \\
\hline 4 & 20,0 & 25,5 & 56,9 \\
\hline 5 & 44,4 & 16,8 & 50,2 \\
\hline 6 & 22,0 & 21,7 & 57,2 \\
\hline 7 & 12,7 & 17,5 & 46,9 \\
\hline 8 & 22,2 & * & 47,4 \\
\hline 9 & 21,4 & * & 51,8 \\
\hline 10 & 40,0 & 30,8 & 53,8 \\
\hline 11 & 33,3 & 42,6 & 53,0 \\
\hline 12 & 7,7 & $*$ & 46,0 \\
\hline 13 & 22,2 & 25,6 & 46,7 \\
\hline 14 & 0,0 & 16,7 & 46,7 \\
\hline 15 & 11,1 & 24,0 & 49,4 \\
\hline$\%$ moyen. & 21,5 & 25,2 & 50,8 \\
\hline
\end{tabular}

* décompte non réalisé

Tableau 1 - Pourcentage de céramique modelée dans le complexe céramique de l'établissement Panskoe I (secteur U7).

ne subsiste principalement que pour les formes servant à la préparation de la nourriture.

La confrontation des résultats des analyses technologiques et morphologiques montre clairement que l'ensemble des objets modelés se divise en trois groupes importants et non équivalents. Le premier et le plus important en nombre d'objets comprend des céramiques qui reproduisent, pour la plupart, des formes scythes ordinaires, caractéristiques à l'époque antique de toute la zone steppique du nord de la mer Noire. Chronologiquement, ce groupe couvre toute la période de l'existence de l'établissement. La forme et l'ornementation, de même que certaines techniques de production caractéristiques des objets du deuxième groupe permettent de le rattacher, dans l'ensemble, à la céramique de la période tardive de la culture de Kizil-Koba. Le troisième groupe de céramique modelée, enfin, est constitué d'objets qui imitent dans une mesure plus ou moins large la céramique tournée grecque.

L'analyse pétrographique apporte la preuve que l'essentiel de cette céramique était produit dans les limites du territoire de l'établissement 
et dans ses environs. Seul pour un groupe relativement peu représenté de céramique modelée de type Kizil-Koba, il faut supposer une production non locale, distante de l'établissement de 30 à $60 \mathrm{~km}$ : il y a été révélé la présence, dans la composition du dégraissant de la pâte argileuse, d'un sable quartzeux absent aux alentours de Panskoe mais caractéristique des plages de la région de la flèche littorale de Bakal et d'Eupatoria. Toutes ces céramiques sont représentées par des fragments plus ou moins grands. Parmi le matériel provenant du bâtiment U6 que j'ai étudié, il n'a été possible de reconstituer aucun exemplaire entier, ce qui distingue également ce groupe des autres variantes de céramique modelée ou tournée. Tout cela montre que sa pénétration sur le territoire de l'établissement s'est faite en une fois au début de la période d'existence du bâtiment U6, suite à une migration, selon toute vraisemblance, plutôt qu'en conséquence d'un commerce régional. Un autre indice de l'affluence d'une nouvelle population à cette époque est la brusque augmentation de la superficie de l'établissement.

Vient ensuite la question de l'interprétation de ces observations. Avant tout, peut-on considérer la présence de céramique modelée, au sein du complexe céramique de l'établissement, comme un témoignage de l'hétérogénéité culturelle et ethnique de la population de Panskoe et de la présence dans l'établissement d'une composante locale non grecque à côté de la paysannerie grecque? Bien que je sois loin de mettre sur un pied d'égalité artefact et ethnos, je continue de croire, comme il a été dit dans ma précédente publication sur les matériaux du bâtiment U6, que l'on peut répondre à cette question par l'affirmative ${ }^{80}$. On en a une remarquable confirmation dans le mobilier de la nécropole, examiné ailleurs

80. Mon interprétation de l'assortiment de céramique modelée du bâtiment U6 a rencontré une objection dans la récente publication de L. Hannestad, «Handmade or Wheel-made». En indiquant, avec raison, l'éventualité de l'usage de céramique modelée par les Grecs, l'auteur ne cherche toutefois pas à expliquer le fait que l'essentiel de cette céramique était produit directement dans l'établissement. Dans l'hypothèse que les Grecs non seulement utilisaient, mais également fabriquaient cette céramique, on n'explique pas pourquoi la préférence pouvait être donnée au moulage primitif à la main au lieu du tour de potier bien connu, et à des formes du répertoire traditionnel des autochtones au lieu de formes plus familières à l'œil grec. Un autre phénomène reste alors inexpliqué, celui de la coexistence de quatre traditions technologiques qui se distinguent par la composition de la pâte céramique, les méthodes de traitement de la surface, les conditions de cuissons et les traditions de décoration. 
en détail ${ }^{81}$, qui permet non seulement de savoir qui étaient les habitants vivant dans l'établissement et quelle était leur apparence extérieure, mais aussi quelles étaient leurs conditions et espérance de vie et quelles étaient les dimensions et structures de leurs maisons d'habitation.

\section{Commerce et échange, circulation monétaire}

Compte tenu de l'éloignement important de Panskoe des principaux centres commerciaux de la polis qu'étaient Chersonèse et Kerkinitis, une partie des opérations commerciales liées à l'écoulement de la production agricole et à l'acquisition de biens de nécessité se déroulait de toute évidence sur le territoire même de l'établissement. C'est aussi là, probablement, que les habitants des petites fermes situées à ses alentours proches convergeaient pour commercer. Seule la vente des céréales, considérées par l'Etat comme une production stratégique, faisait l'objet de règles particulières, même si tel n'était apparemment pas toujours le cas. On trouve une indication de cela dans le texte du fameux serment de Chersonèse (IOSPE $\left.\mathrm{I}^{2}, 401\right)$, publié à la fin du $\mathrm{IV}^{\mathrm{e}}$ siècle ou au tournant des $\mathrm{IV}^{\mathrm{e}}$ et III ${ }^{\mathrm{e}}$ s. av. J.-C., au moment où la ville traversait de sérieuses perturbations politiques et sans doute économiques. Les lignes 47-50 de ce document interdisent la vente et le transport de céréales produites «en plaine» (par quoi il faut comprendre le territoire du nord-ouest de la Crimée) vers quelque endroit que ce soit autre que Chersonèse. J'ai examiné en détail dans une publication consacrée à ces questions les raisons qui ont poussé les autorités de la ville à inclure ce paragraphe dans le texte du document ${ }^{82}$, ce qui nous acquitte de la nécessité de nous y arrêter encore une fois ici.

En échange de céréales et de produits de l'élevage, les périphéries rurales de l'Etat de Chersonèse accédaient à du vin d'importation (en provenance d'Héraclée du Pont, de Sinope, de Thasos, de Chios, de Mendè ou d'autres lieux) ou produit à Chersonèse, à de l'huile d'olive,

8I. V. F. Stolba, «Multicultural Encounters in the Greek Countryside»; V. F. Stolba, E. Rogov, Panskoye I, vol. 2.

82. V. F. Stolba, "The Oath of Chersonesos and the Chersonesean Economy in the Early Hellenistic Period». 
des olives salées ou marinées ${ }^{83}$, des objets de verre et de métal, ainsi qu’à un large assortiment d'objets de céramique. Avec le passage du nordouest de la Crimée sous la juridiction de Chersonèse, la production des ateliers de céramique chersonésites domine nettement dans les trouvailles locales. Le pourcentage de céramique attique à vernis noir et de ses imitations est également élevé, ainsi que celui de céramique dite d'argile grise ${ }^{84}$, produite à Olbia et dans d'autres centres du nord de la mer Noire, y compris à Chersonèse. Une part importante de la production de centres étrangers qui parvenait jusque-là, sans doute pas directement mais via Chersonèse, faisait de toute évidence partie elle aussi du cycle commercial régional ${ }^{85}$. Kerkinitis et Olbia pouvaient constituer d'autres centres comparables de distribution, surtout au début du IV s. av. J.-C. $\mathrm{Si}$ l'on en juge par le fait que des monnaies de ces deux villes se retrouvent à plusieurs reprises dans les trouvailles monétaires de Panskoe, les liens économiques avec ces centres subsistent à la fin du IVe siècle.

Une analyse comparative des marques de vente et des signes de propriété sur les amphores trouvées dans le bâtiment U6 de l'établissement de Panskoe permet d'arriver à cette très importante conclusion: dans le domaine du commerce du vin, une des variétés de transaction de vente qui a pu avoir cours dans la chôra de Chersonèse a été manifestement la vente sur commande préalable ${ }^{86}$. On ignore si cette dernière touchait aussi les autres marchandises. Il ne fait en revanche pas de doute que le commerce de détail était également pratiqué dans l'établissement. Les trouvailles de petites monnaies d'échange et de poids de balance en plomb, qui correspondaient aux différentes fractions de la mine grecque, en sont des témoignages révélateurs. Dans le cas d'une trouvaille, il s'agit d'une pièce de monnaie en bronze d'Olbia, dite "borysthène", dont les

83. Trois noyaux intacts d'olive de culture ont été découverts à Panskoe I, datant du dernier quart du IV e s. av. J.-C. Voir A. N. Ščeglov et al., "Zemledelie na poselenii Panskoe I (Severo-Zapadnyj Krym) v IV - načale III v. do n.è.», p. 64 sq. A en juger par les renseignements donnés par les voyageurs des XVIII et XIX ${ }^{\mathrm{e}}$ siècles, les oliviers ont été cultivés en Crimée méridionale jusqu’à une période récente. On ignore si cette culture était pratiquée sur la presqu'île de Tarkhankut dans l'Antiquité ou à des périodes plus récentes.

84. Voir S. Handberg, V. F. Stolba, S. V. Ušakov, «Classical and Hellenistic Grey Ware from the Western Crimea".

85. L. Hannestad, " "How much came from where” ", p. 182.

86. V. F. Stolba, «Handmade Pottery»; "Kauri i pročie amulety v pogrebal'nom obrjade nekropolja Panskoe I». 
bords ont été limés pour ajuster sa masse afin de l'utiliser comme poids de mesure.

Alors que le troc devait jouer et jouait assurément un rôle important à toutes les étapes de l'histoire de la chôra de Chersonèse, la monétisation du marché rural et le passage à des formes monétaires de règlement, surtout dans les établissements de la zone littorale, s'effectuent pratiquement simultanément à l'assimilation par les Chersonésites de la Tauride occidentale. Au cours de toutes les années de fouilles et de collectes sur le site de Panskoe I, il a été trouvé 75 exemplaires de pièces de monnaie ${ }^{87}$, ce qui représente une collection que seules les trouvailles de Chersonèse et de Kerkinitis dépassent en nombre. Parmi ces pièces, 54 exemplaires, soit $72 \%$, sont précisément des pièces de Chersonèse. Les plus anciennes d'entre elles, tant à Panskoe que dans d'autres sites ruraux de la région, datent d'environ 360 av. J.-C. ${ }^{88}$. S'appuyant sur la chronologie traditionnelle, aujourd'hui obsolète, qui fait remonter la colonisation de la presqu'île de Tarkhankut par Chersonèse à la deuxième moitié du siècle, on a pu faire des tentatives de voir dans ces trouvailles le résultat de contacts commerciaux précoces, ou une ancienne monnaie qui aurait dû être frappée longtemps avant que les colons chersonésites ne l'introduisent dans ces régions ${ }^{89}$. Ces explications sont cependant invalidées par le fait que la plupart de ces trouvailles sont des pièces de bronze de très faible valeur nominale, impropres au commerce international ou lointain, et par le fait également qu'elles sont trop nombreuses pour être considérées comme fortuites. Il ne fait actuellement plus de doute que

87. Dans une précédente publication portant sur la découverte de monnaies en provenance de l'établissement, A. M. Gilevič, ("Osobennosti denežnogo rynka poselenija Panskoe I (Severo-Zapadnyj Krym) v IV-III vv. do n.è.», p. 12) n'en mentionne que 63 exemplaires. Je dois à I. Mirončuk et à V. B. Užencev l'information sur les 12 autres pièces de monnaie, conservées dans des collections particulières et provenant de collectes illégales à l'aide d'un détecteur de métaux.

88. A. M. Gilevič, "Khersones i Severo-Zapadnyj Krym po numizmatičeskim dannym», p. 55; V. F. Stolba, "Dom IV v. do n.è. na poselenii Panskoe I», p. 80; S. A. Kovalenko, "Nakhodki monet s gorodišča Čajka v Severo-Zapadnom Krymu», p. 145, fig. 2 et 149, n os 3-4; V. A. Kutajsov, V. B. Užencev, «Issledovanija gorodišča Kalos Limena», p. 87. Seule une trouvaille remonte à une période plus ancienne, une monnaie coulée d'Istros trouvée sur l'établissement Panskoe I. Voir A. M. Gilevič, V. F. Stolba, A. N. Ščeglov, «Nakhodka monety Istrii v Severo-Zapadnom Krymu».

89. S. Ju. Saprykin, Denežnoe obraščenie na khore Khersonesa Tavričeskogo v antičnuju èpokhu, p. 45. 
la monnaie chersonésite arrive dans cette région en même temps que les colons de Chersonèse. Dans la deuxième moitié du IV ${ }^{\mathrm{e}}$ s. av. J.-C., des monnaies de bronze de Kerkinitis et de Panticapée, découvertes dans toute une série d'établissements, font également partie de la circulation monétaire de la chôra lointaine de Chersonèse. A Panskoe, leur pourcentage se monte respectivement à $12 \%$ et $8 \%$. Des monnaies d'autres villes ont été trouvées sur l'établissement, avec des exemplaires d'Histria, Olbia ou Larissa en Thessalie ${ }^{90}$. A la différence toutefois de celles de Kerkinitis ou de Panticapée, ces pièces sont isolées; elles ne faisaient certainement pas partie de la circulation monétaire sur le marché local, mais elles attestent de l'existence de contacts entre l'établissement et ces centres lointains.

Les trouvailles monétaires sur le territoire de Tarkhankut, datées tant de l'étape la plus ancienne que des époques ultérieures, sont représentées presque exclusivement par des pièces en bronze, qui pouvaient souvent rester en circulation plus longtemps qu'en ville ${ }^{91}$. Quelques trésors de petite taille et trouvailles collectives faites sur le territoire de Crimée occidentale (trésor de Kučuk-Mojnak, trésor de l'établissement de Majak, trésor d'Eupatoria de 1941, ainsi que des trouvailles de Panskoe et de Vladimirovka) ${ }^{92}$, ne présentent eux aussi que des pièces de cuivre. Des découvertes de pièces d'argent en dehors de Chersonèse et de Kerkinitis sont très rares, bien qu'il y en ait eu. Une trouvaille unique d'une drachme de Chersonèse de la fin du IV ${ }^{\mathrm{e}}$ ou du début du III ${ }^{\mathrm{e}}$ s. av. J.-C., présentant la tête d'Héraclès sur l'avers et un taureau chargeant sur le revers, a été attestée sur le site de Južno-Donuzlavskoe ${ }^{93}$. Une autre pièce analogue, portant des contremarques des deux côtés, a été découverte en 1989 sur le site de Čajka ${ }^{94}$. Enfin, la découverte près

90. A. M. Gilevič, «Moneta Larissy iz poselenija Panskoe I».

9I. Comparer avec A. M. Gilevič, "Khersones i Severo-Zapadnyj Krym po numizmatičeskim dannym», p. 55; "Osobennosti denežnogo rynka poselenija Panskoe I (Severo-Zapadnyj Krym) v IV-III vv. do n.è.", p. 13.

92. A. B. Kolesnikov, "Monety iz raskopok usadeb u Evpatorijskogo majaka", p. 186 sq. et 191-193; A. M. Gilevič, "Kučuk-Mojnakskij klad khersonesskikh monet IV-II vv. do n.è.»; "Khronologija i topografija kladov i kollektivnykh nakhodok khersonesskikh monet IV - II vv. do n.è.", p. $363, \mathrm{n}^{\text {o }}$ I.4 et 365 sq., $\mathrm{n}^{\text {os }}$ II.5-8.

93. V. F. Stolba, A. S. Golencov, «Monety iz raskopok i slučajnykh nakhodok na Južno-Donuzlavskom gorodišče», p. 275, n ${ }^{\circ} 1$ et 277 , fig. 1 .

94. De type Anokhin 114. Voir V. F. Stolba, A. S. Golencov, «Monety iz raskopok i slučajnykh nakhodok na Južno-Donuzlavskom gorodišče», p. 276; S. A. Kovalenko, 
du site de Kara-Tobe d'un didrachme de Chersonèse de type "tête de la déesse Parthénos - Parthénos terrassant une biche» (Anokhin 88-90), datée du dernier tiers du IV ${ }^{\mathrm{e}}$ s. av. J.-C. ${ }^{95}$, est bien connue. Un peu plus au sud, au-delà du territoire de Tarkhankut, des monnaies d'argent de Chersonèse ont été découvertes dans le petit trésor malheureusement perdu de Novo-Fedorovka ${ }^{96}$. Je viens en outre d'apprendre la trouvaille fortuite dans l'établissement fortifié de Kunan d'une drachme d'argent de Chersonèse du milieu du $\mathrm{II}^{\mathrm{e}}$ s. av. J.-C. ${ }^{97}$. Ainsi, malgré la rareté de ces trouvailles, il est prématuré de tirer la conclusion que la monnaie d'argent était absente de la circulation sur le territoire de Tarkhankut et que toutes les opérations monétaires sur le marché local se limitaient exclusivement aux petites pièces d'échange en bronze.

\section{Religion et culture}

Malgré l'éloignement des centres urbains, qui étaient aussi en général les centres religieux et cultuels de l'Etat, la population rurale de la chôra lointaine de Chersonèse avait une activité religieuse bien vivante, même si on observe distinctement dans ces régions toute une série d'intéressantes particularités.

Il n'est guère étonnant, compte tenu du caractère économique de l'établissement de Panskoe et de son éloignement des remparts d'une ville à l'intérieur de laquelle les habitants auraient pu se réfugier en cas de danger, que les principales divinités vénérées par la paysannerie locale aient été Héraclès, protecteur et défenseur du territoire de Chersonèse, ainsi que les divinités associées à l'agriculture. Cette particularité est tout

«Nakhodki monet s gorodišča Čajka v Severo-Zapadnom Krymu», p. 148 et 150, $\mathrm{n}^{\mathrm{o}} 36$, fig. 2.1 .

95. Je remercie I. Mirončuk pour cette information.

96. A. M. Gilevič, "Khronologija i topografija kladov i kollektivnykh nakhodok khersonesskikh monet IV - II vv. do n.è.", p. 369, n ${ }^{\circ}$ V.2 ; V. F. Stolba, A. S. Golencov, "Monety iz raskopok i slučajnykh nakhodok na Južno-Donuzlavskom gorodišče", p. 276 , n. 6 .

97. Au sujet de l'établissement de Kunan, voir T. N. Smekalova, V. F. Stolba, Materials for the Archaeologocal Map of Crimea, p. 12 et 51-54, fig. 29-32. La monnaie d'argent, avec quelques autres de bronze, a été découverte en 2010 au cours d'une recherche illégale sur le territoire avec un détecteur de métaux. 
aussi caractéristique des autres établissements ruraux de la Tauride du nord-ouest.

Malgré ses dimensions considérables, Panskoe ne disposait apparemment pas d'un complexe cultuel unique qui aurait répondu aux besoins de toute la communauté. Les sanctuaires domestiques figuraient le lieu principal du culte rendu aux différentes divinités. Deux sanctuaires de ce type ont été étudiés en détail au cours de fouilles sur l'établissement. Le plus grand des deux, découvert dans la pièce $12 \mathrm{du}$ bâtiment U6 ${ }^{98}$, était consacré à Déméter et à Sabazios, ce qu'attestent de nombreuses terres cuites, ainsi que l'inscription dédicatoire i $\varepsilon \rho \grave{\alpha}$ $\Sigma \alpha \beta \alpha \zeta i o v$ sur la paroi d'un skyphos à vernis noir ${ }^{99}$, inscription qui est le plus ancien témoignage du culte de Sabazios non seulement sur le territoire de l'Etat de Chersonèse, mais aussi dans tout le bassin de la mer Noire. Dans une pièce indépendante, devant l'entrée de ce sanctuaire, il y avait un sanctuaire à Héraclès où l'on trouvait, outre un autel de pierre, un relief de calcaire fixé au mur et représentant le héros ${ }^{100}$. Ce genre de reliefs, qui montrent un Héraclès debout ou couché et qui sont réalisés avec plus ou moins de maîtrise, sont caractéristiques des établissements ruraux de la chôra lointaine de Chersonèse: on les rencontre sur bien des sites (Mežvodnoe, Kul'čuk, Mojnaki, Čajka, Saki). A en juger par un graffito dédicatoire sur la paroi d'un très grand canthare, le sanctuaire domestique découvert à Panskoe, dans le bâtiment 15 du secteur U7, était également consacré à Héraclès Sôter ${ }^{101}$. Dans ce même bâtiment a été découverte une statuette en terre cuite de Pan, considéré comme le protecteur des bergers et de l'élevage (fig. 9). A côté du culte d'Héraclès, on célébrait peut-être à Panskoe le culte de quelque héros local; c'est selon toute vraisemblance ce héros que représente la statuette composite de calcaire mentionnée plus haut, qui représente un guerrier en pied sur un socle, le bras droit replié, une coupe à la main et qui prend appui de la main gauche sur un bouclier circulaire. Parmi les autres divinités,

98. A. Chtcheglov, Polis et chora, p. 173-175; A. N. Ščeglov, «Monumental Building U6», p. 45-50.

99. V. F. Stolba, «Graffiti and Dipinti», p. 229, H 2, pl. 150, 156.

IOo. A. N. Ščeglov, Severo-Zapadnyj Krym v antičnuju èpokhu, p. 124, fig. 66.1; "Cult sculpture, Altars, Sacred Vessels and Votivs", p. 213 et 221, G 1, pl. 143; "Monumental Building U6», p. 51.

IOI. V. F. Stolba, «Novoe posvjaščenie iz Severo-Zapadnogo Kryma i aspekty kul'ta Gerakla v Khersonesskom gosudarstve». 


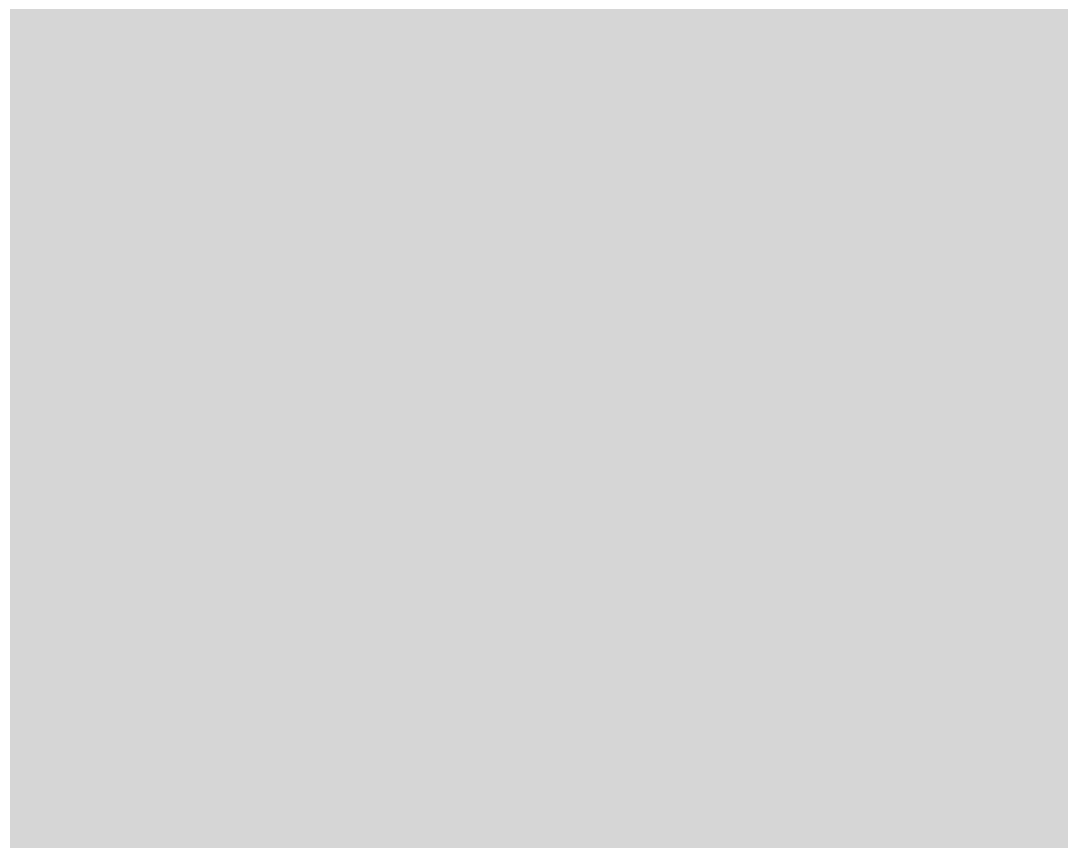

Fig. 9 - Statuettes en terre cuite provenant des fouilles du secteur central U7.

on trouve également des traces dans l'établissement d'une vénération pour Aphrodite. Outre les protomés en terre cuite découverts dans bon nombre de complexes, on trouve de cela un témoignage révélateur dans l'inscription dédicatoire 'A $\phi \rho[0] \delta i ́ \tau \alpha[1 / \varsigma$, entaillée sur la paroi d'un canthare attique à vernis noir ${ }^{102}$.

Incontestablement, le culte des morts occupait lui aussi une place importante dans la vie spirituelle de la population locale: des libations aux défunts étaient annuellement organisées sur des tables sacrificielles installées près des crépis entourant les kourganes qui servaient de sépultures familiales. Pour ceux qui étaient morts en mer ou loin de leur patrie, et dont le corps ne pouvait pas être inhumé, on construisait des cénotaphes, dont un (K32 M1) a été exploré dans la nécropole de Panskoe. Peut-être s'agit-il précisément du cénotaphe ( $\psi \varepsilon] v \delta \alpha ́ p t o v)$ dont il est question dans une lettre particulière sur céramique du complexe U7, où il est demandé de déposer sur ce dernier des sacrifices aux

IO2. U7/79, liste d'enregistrement 120/193; non publié. 
Fig. 10 - Graffiti de l'établissement (1, 3-4) et de la nécropole (2).

moires ${ }^{103}$. Les paysans locaux étaient également familiers des superstitions religieuses, dont la plus persistante était la croyance en le mauvais œil ${ }^{104}$, répandue chez presque tous les peuples et à toutes les époques.

Une collection impressionnante de graffiti, qui compte plus de deux cents inscriptions sur céramique, dans lesquelles le dialecte dorique règne sans partage dès la deuxième moitié $\mathrm{du} \mathrm{IV}^{\mathrm{e}} \mathrm{s}$. av. J.-C., témoigne d'un niveau assez élevé d'alphabétisation de l'établissement de Panskoe (fig. 10). Les noms propres qui y sont représentés sont exclusivement d'origine grecque ou d'Asie Mineure. Cela ne peut toutefois en aucun cas servir de preuve de l'absence sur l'établissement d'une composante barbare, probablement analphabète et restée pour cette raison dans l'ombre de l'histoire.

I03. V. F. Stolba, "Grečeskoe pis'mo s poselenija Panskoe I (Severo-Zapadnyj Krym)»; Bull. ép. 2006, 295; SEG LV 859.

I04. V. F. Stolba, «Kauri i pročie amulety v pogrebal'nom obrjade nekropolja Panskoe I», p. 157-160; «Busy, podveski i amulety». 


\section{Conclusion}

Les matériaux archéologiques dégagés au cours des prospections archéologiques sur l'établissement de Panskoe I et dans ses environs nous permettent ainsi de brosser un tableau haut en couleur de la vie rurale d'une population à la périphérie de la polis grecque qu'était Chersonèse Taurique. Il reste cependant de nombreuses questions de fond (concernant par exemple le caractère de la propriété terrienne, le statut et l'organisation sociale de la population, le pourcentage en son sein de population non grecque, les forces de travail, etc.) auxquelles nos sources ne nous permettent pas encore de répondre. La grande distance séparant Panskoe de Chersonèse, le caractère des constructions d'habitation et la présence d'une vaste nécropole comptant une grande quantité d'inhumations d'enfants et de familles, tout cela ne permet pas de douter que l'essentiel de la population y demeurait toute l'année, même si des vents extraordinairement forts et des températures hivernales relativement basses mettaient cette population à rude épreuve. A la différence de Panskoe, où le choix de l'emplacement de l'établissement fut dicté par des considérations stratégiques (la présence d'un port maritime ainsi que le fort préexistant de l'époque olbienne), les petits hameaux et villages, qui surgirent à nouveau durant la deuxième moitié du IVe s. av. J.-C. et que nos prospections de 2009-2011 ont permis de découvrir en grand nombre dans les régions reculées de la presqu'île, tenaient compte pour s'établir du facteur climatique et s'adaptaient de fait aux particularités du paysage.

La principale spécialisation économique de l'établissement était l'agriculture, et avant tout la culture de céréales panifiables. A côté du blé et de l'orge traditionnels, le seigle apparaît au début du III ${ }^{\mathrm{e}}$ s. av. J.-C. en tant que semence autonome. Miser sur le seigle, céréale non commerçable mais plus résistante et garantissant une récolte même durant les années maigres, était manifestement la réaction de la paysannerie locale à la détérioration des conditions climatiques de la région. La viticulture et l'horticulture, bien que pratiquées dans l'établissement, n'avaient pas l'importance qu'elles pouvaient avoir dans les établissements des alentours immédiats de Chersonèse, sur la presqu'île d'Héraclée. Malgré des trouvailles de noyaux d'olives, base avec les céréales et le raisin de la triade méditerranéenne, cette culture n'était assurément pas pratiquée à cet endroit, où la place de l'olivier était occupée par les légumineuses. 
Dans l'économie de l'établissement, incontestablement, un rôle important était dévolu à l'élevage, représenté surtout par l'élevage ovin, ce qui permet de la qualifier d'économie agro-pastorale plutôt que purement agricole. La pêche, si elle était un appoint alimentaire substantiel pour les habitants, n'avait probablement pas de véritable portée économique. Une grande quantité de trouvailles de pièces de monnaie, y compris étrangères, témoigne par ailleurs de l'importance de l'établissement comme port maritime et centre de commerce de détail.

La Crimée du nord-ouest reste le principal grenier de Chersonèse jusqu'en 270 av. J.-C., époque où suite à un changement climatique et sous la pression des nomades, le territoire agricole de la polis se réduisit au minimum et son économie subit un profond déclin. Le dernier tiers du siècle connut une renaissance de courte durée de la chôra, mais ce ne fut qu'un bref répit sur la voie de décadence définitive de Chersonèse comme centre économique et politique majeur de la région du Pont septentrional.

Vladimir Fjodorovič STOLBA Université d'Aarhus

Avec le soutien du Danish Council for Independent Research (bourse 09-069235)

Traduit du russe par Mathilde ReICHLER IMPeriali 


\section{BIBLIOGRAPHIE}

\section{Abréviations}

Bull. ép. = Bulletin épigraphique, dans Revue des études grecques, Paris, 1888 -

$I G=$ Inscriptiones Graecae, Berlin, 1903-

$I O S P E=$ LATYŠEv, B., Inscriptiones antiquae orae septentrionalis Ponti Euxini Graecae et Latinae, Saint-Pétersbourg, 1885-1901.

SEG $=$ Supplementum Epigraphicum Graecum, Leyde, 1923-1971, puis Amsterdam, 1979-

\section{Etudes}

Alcock, Susan E., Gates, Jennifer E., Rempel, Jane E., «Reading the Landscape: Survey Archaeology in the Hellenistic Oikumene», in A Companion to the Hellenistic World, ed. by Andrew Erskine, Oxford, Blackwell Publishing, 2005, p. 354-372.

Alcock, Susan E., Rempel, Jane E., "The More Unusual Dots on the Map: "Special-Purpose" Sites and the Texture of Landscape", in Surveying the Greek Chora. The Black Sea Region in a Comparative Perspective, ed. by Pia Guldager Bilde, Vladimir F. Stolba, Aarhus, University Press, 2006, p. 27-46.

Anfimov, Nikita Vladimirovič, "Skotovodstvo u sindo-meotskikh plemjon Prikuban'ja» [L'élevage chez les tribus sindo-méotes du Kouban»], Naučnye trudy Krasnodarskogo gosudarstvennogo pedagogičeskogo instituta, 103 (1969), p. 3-17.

Anokhin, Vladilen Afanas'evič, Monetnoe delo Khersonesa (IV v. do n.è. - XII v. n.è.) [Le monnayage de Chersonèse Taurique (IVe siècle avant notre ère - XII e siècle de notre ère)], Kiev, Naukova Dumka, 1977. 
Bilde, Pia Guldager, Attema, Peter, Lancov, Sergej Borisovič, Smekalova, Tatiana Nikolaevna, Stolba, Vladimir Fjodorovič, Haas, Timon de, Handberg, Søren, Jakobsen, Kristina W., «Džarylgač Survey Project (DSP). The Results of Campaign of 2007 ", in Bosporskij fenomen. Sakral'nyj smysl regiona, pamjatnikov, nakhodok. Materialy meždunarodnoj naučnoj konferencii [Le phénomène bosporan. Signification sacrée de la région, des monuments, des trouvailles. Actes du colloque scientifique international], otv. red. Vadim Jur'evič Zuev et al., Sankt-Peterburg, 2007, p. 107-118 et 376-377.

Bilde, Pia Guldager, Stolba, Vladimir F. (eds), Surveying the Greek Chora. The Black Sea Region in a Comparative Perspective, Aarhus, University Press, 2006.

Blagovolin, Nikolai S., Ščeglov, Alexander N., "Archaeological, Paleogeographic, and Geomorphological Researches in the Lake Sasyk (Panskoye) Region", in Panskoye I, vol. I: The Monumental Building U6, ed. by Lise Hannestad, Vladimir F. Stolba, Alexander N. Ščeglov, Aarhus, University Press, 2002, p. 285-302.

Brendel, Otto, Die Schafzucht im alten Griechenland, Würzburg, Triltsch, 1934.

Bujsкiкh, Alla Valer'evna, Prostranstvennoe razvitie Khersonesa Tavričeskogo $v$ antičnuju èpokhu [Le développement spatial de Chersonèse Taurique durant l'Antiquité], Simferopol', Demetra, 2008.

Calkin, Veniamin Iosifovič, "Domašnie i dikie životnye Severnogo Pričernomor’ja v èpokhu rannego železa» "Animaux domestiques et sauvages du littoral septentrional de la mer Noire à l'âge du fer récent»], Materialy $i$ issledovanija po arkheologii SSSR, 53 (1960), p. 7-109.

Cartledge, Paul, «Classical Greek agriculture: Recent work and alternative views", Journal of Peasant Studies, 21/1 (1993), p. 127-136.

-, "The economy (economies) of ancient Greece», in The Ancient Economy, ed. by Walter Scheidel, Sitta von Reden, Edinburgh, University Press, 2002, p. 11-32.

Cherry, John F., "Regional Survey in the Aegean: The "New Wave" (and After)", in Beyond the Site. Regional Studies in the Aegean 
Area, ed. by Paul N. Kardulias, Lanham/New York/London, University Press of America, 1994, p. 91-112.

—, "Archaeology Beyond the Site: Regional Survey and its Future», in Theory and Practice in Mediterranean Archaeology: Old World and New World Perspectives, ed. by John K. Papadopoulos, Richard M. Leventhal, Los Angeles, Cotsen Institute of Archaeology, University of California, 2003, p. 137-159.

Chtcheglov, Alexander, Polis et chora. Cité et territoire dans le PontEuxin, Paris, Les Belles-Lettres, 1992.

Currid, John D., Navon, Avi, «Iron Age Pits and the Lahav (Tell Halif) Grain Storage Project», Bulletin of the American Schools of Oriental Research, 273 (1989), p. 67-78.

DAšEvskaja, Olga Davydovna, «Antičnaja bašnja na gorodišče Beljaus» ["Une tour antique sur le site de Beljaus»], Kratkie soobščenija instituta arkheologii, 116 (1969), p. 85-92.

Diatroptov, Pavel Danilovič, "Pifosy s gorodišča Beljaus s graffiti » " Des pithoi avec graffiti provenant du site de Belajus»], Arkheologija, 1 (1999), p. 177-183.

Finley, Moses, "The City», Opus 6-8 (1987-1989), p. 303-315.

Gallant, Thomas W., Risk and Survival in Ancient Greece, Cambridge Polity Press, 1991.

Gilevič, Anna Michajlovna, "Kučuk-Mojnakskij klad khersonesskikh monet IV-II vv. do n.è.» ["Trésor de monnaies chersonésites du $\mathrm{IV}^{\mathrm{e}}$ au II ${ }^{\mathrm{e}}$ siècle avant notre ère découvert à Kučuk-Mojnak»], Numizmatika i èpigrafika, 8 (1970), p. 3-16.

—, «Khersones i Severo-Zapadnyj Krym po numizmatičeskim dannym " [Chersonèse et la Crimée du nord-ouest à la lumière de la numismatique»], in Novoe $v$ sovetskoj numizmatike $i$ numizmatičeskom kraevedenii (K 200-letiju Otdela numizmatiki Ermitaža) [Nouveautés en numismatique soviétique et en numismatique régionale (A l'occasion du bicentenaire du département de numismatique de l'Ermitage)], Leningrad, 1987, p. 54-55.

—, «Moneta Larissy iz poselenija Panskoe I» [ Une monnaie de Larissa provenant de l'établissement de Panskoe I"], in Drevnee Pričernomor'e. Tezisy dokladov [Le littoral pontique ancien. Résumés des conférences], Odessa, 1989, p. 19-20.

-, «Osobennosti denežnogo rynka poselenija Panskoe I (SeveroZapadnyj Krym) v IV-III vv. do n.è.» [Spécificités du marché 
monétaire de l'établissement de Panskoe I (Crimée du nordouest) au IV ${ }^{\mathrm{e}}$ et III ${ }^{\mathrm{e}}$ siècle avant notre ère»], in $I V$ Vserossijskaja numizmatičeskaja konferencija $v$ g. Dmitrove, 22-26 aprelja 1996 g. [IV Conférence russe de numismatique à Dmitrov, 22-26 avril 1996], otv. red. Aleksander Stepanovič Beljakov, Moskva, 1996, p. 12-13.

—, «Khronologija i topografija kladov i kollektivnykh nakhodok khersonesskikh monet IV - II vv. do n.è.» " "Chronologie et topographie des trésors et trouvailles collectives de monnaies chersonésites du $\mathrm{IV}^{\mathrm{e}}$ au II $\mathrm{II}^{\mathrm{e}}$ siècle avant notre ère»], Arkheologičeskie Vesti, 6 (1999), p. 346-373.

Gilevič, Anna Mikhajlovna, Stolba, Vladimir Fjodorovič, Ščeglov, Aleksander Nikolaevič, "Nakhodka monety Istrii v SeveroZapadnom Krymu» ["Découverte d'une monnaie d'Histria en Crimée du nord-ouest»], in Drevnee Pričernomor'e [Le littoral pontique ancien], II, Odessa, 1991, p. 22-23.

Handberg, Søren, Stolba, Vladimir F., Ušakov, Sergej V., "Classical and Hellenistic Grey Ware from the Western Crimea", in Pontic Grey Wares, ed. by Pierre Dupont, Vasilica Lungu, Constanţa, Muzeul de Istorie Naţională şi Arheologie Constanţa, 2009, p. 167-185 (Pontica XLII. Suppl. 1).

Hannestad, Lise, "How much came from where": The proportion of local, regional and "long-distance" pottery from a rural settlement in the Crimea", in Making, Moving and Managing. The New World of Ancient Economies, 323-31 BC, ed. by Zofia G. Archibald, John K. Davies, Vincent Gabrielsen, Oxford, Oxbow, 2005, p. 165-187.

-, "The Dating of the Monumental Building U6 at Panskoe I», in Chronologies of the Black Sea Area in the Period c. 400-100 BC, ed. by Vladimir F. Stolba, Lise Hannestad, Aarhus, University Press, 2005, p. 179-192.

—, «Handmade or Wheel-made: a Note on the Issue of a Northern Pontic Cultural koinè», in Une koinè pontique. Cités grecques, sociétés indigènes et empires mondiaux sur le littoral nord de la mer Noire (VII ${ }^{e}$. a. C. $-I I I^{e}$ s. p. C.), éd. par Alain Bresson, Askold Ivantchik, JeanLouis Ferrary, Bordeaux, Ausonius, 2007, p. 141-147.

Hannestad, Lise, Stolba, Vladimir F., Blinkenberg Hastrup, Helene, "Black-Glazed, Red-Figure and Grey Ware Pottery», 
in Panskoye I, Vol. 1: The Monumental Building U6, ed. by Lise Hannestad, Vladimir F. Stolba, Alexander N. Ščeglov, Aarhus, University Press, 2002, p. 127-149.

Hannestad, Lise, Stolba, Vladimir F., Ščeglov, Alexander N. (eds), Panskoye I, Vol. 1: The Monumental Building U6, Aarhus, University Press, 2002.

Hansen, Mogens Herman, The Shotgun Method. The Demography of the Ancient Greek City-State Culture, Columbia/London, University of Missouri Press, 2006.

Hanson, Victor Davis, The Other Greeks. The Family Farm and the Agrarian Roots of Western Civilization, New York, The Free Press, 1995.

Hodkinson, Stephen J., "Animal Husbandry in the Greek Polis», in Pastoral Economies in Classical Antiquity, ed. by Charles R. Whittaker, Cambridge, Cambridge Philological Society, 1988, p. 35-74.

Isager, Signe, Skydsgaard, Jens E., Ancient Greek Agriculture, London, Routledge, 1992.

JACENKo, Irina Vladimirovna, «Vinodel'nja na poselenii Čajka» [ Un atelier viticole sur l'établissement de Čajka»], Kratkie soobščenija instituta arkheologii, 174 (1983), p. 18-25.

JANuŠEvič, Zoja Vasil'evna, Kul'turnye rastenija Jugo-Zapada SSSR po paleobotaničeskim issledovanijam [Plantes cultivées de la partie sud-ouest de l'URSS à la lumière des recherches paléobotaniques], Kišinev, Štiinca, 1976.

—, «Die Kulturpflanzen Skythiens», Zeitschrift für Archäologie, 15 (1981), p. 87-96.

—, Kul'turnye rastenija Severnogo Pričernomor'ja. Paleobotaničeskie issledovanija [Plantes cultivées du littoral septentrional de la mer Noire. Recherches paléobotaniques], Kišinev, Štiinca, 1986.

Januševič, Zoya V., Ščeglov, Alexander N., «Palaeoethnobotanical Material", in Panskoye I, Vol. 1: The Monumental Building U6, ed. by Lise Hannestad, Vladimir F. Stolba, Alexander N. Ščeglov, Aarhus, University Press, 2002, p. 327-331.

Kasparov, Aleksei K., "Osseous Remains", in Panskoye I, Vol. 1: The Monumental Building U6, ed. by Lise Hannestad, Vladimir F. Stolba, Alexander N. Ščeglov, Aarhus, University Press, 2002, p. 332-333. 
Khrapunov, Igor Nikolaevič, Smekalova, Tatiana Nikolaevna, Stolba, Vladimir Fjodorovič, "Razvedki 2009 g. v Černomorskom r-ne Avtonomnoj Respubliki Krym» ["Prospections réalisées en 2009 dans le district de Černomorskoe en République autonome de Crimée "], in Arkheologični doslidžennja v Ukraïni 2009 [Recherches archéologiques en Crimée durant l'année 2009], Kyïv-Luc'k, Volyns'ki starožytnosti, 2010, p. 452-453.

Kolesnikov, Andrej Borisovič, "Monety iz raskopok usadeb u Evpatorijskogo majaka» ["Monnaies issues des fouilles de la propriété proche du phare d'Eupatoria»], in Pamjatniki železnogo veka v okrestnostjakh Evpatorii [Monuments de l'âge du fer des alentours d'Eupatoria], otv. red. Julija Leonidovna Ščapova, Irina Vladimirovna Jacenko, Moskva, Izdatel'stvo MGU, 1991, p. 186-195.

Kolesnikov, Andrej Borisovič, Jacenko, Irina Vladimirovna, «Le territoire agricole de Chersonèse taurique dans la région de Kerkinitis", in Territoires des cités grecques, éd. par Michèle Brunet, Paris, De Boccard, 1999, p. 289-321 (BCH Suppl. 34).

Kovalenko, Sergej Anatol'evič, «Nakhodki monet s gorodišča Čajka v Severo-Zapadnom Krymu (1959-2002 gg.) » [ ¿écouvertes monétaires du site de Čajka en Crimée du nord-ouest (années 1959 à 2002)»], Problemy istorii, filologii, kul'tury, Moskva-Magnitogorsk, 16 (2006), p. 144-154.

Kruglikova, Irina Timofeevna, Sel'skoe khozjastvo Bospora [L'agriculture du Bosphore], Moskva, Nauka, 1975.

Kutajsov, Vadim Aleksandrovič, "Agrarnaja istorija Kerkinitidy (I)" ["Histoire agraire de Kerkinitis (I)»], Khersonesskij Sbornik, 10 (1999), p. 83-93.

Kutajsov, Vadim Aleksandrovič, Užencev, Vjačeslav Borisovič, «Issledovanija gorodišča Kalos Limena» ["Investigations sur le site de Kalos Limèn"], in Arkheologičeskie issledovanija v Krymu 1995 [Recherches archéologiques en Crimée en 1995], Simferopol', Sonat, 2007, p. 87-91.

Liberov, Pjotr Dmitrievič, "K istorii skotovodstva i okhoty na territorii Severnogo Pričernomor’ja v èpokhu rannego železa» [ "De l'histoire de l'élevage et de la chasse sur le territoire du Pont septentrional à l'âge du fer récent»], Materialy $i$ issledovanija po arkheologii SSSR, 53 (1960), p. 110-164. 
Longhurst, Richard, "Household Food Strategies in Response to Seasonality and Famine", Institute of Development Studies Bulletin, 17/3 (1986), p. 27-35.

Marčenko, Konstantin K., Žıtnikov, Viktor G., Kopylov, Viktor P., Die Siedlung Elizavetovka am Don, Mainz, von Zabern, 2000.

Michell, Humfrey, The Economics of Ancient Greece, Cambridge, W. Heffer, $1963^{2}$.

Neustrueva, Irina Jur'evna, Subetto, Dmitrij Aleksandrovič, Stolba, Vladimir Fjodorovič, SApelko, Tatiana Valentinovna, Kuznecov, Denis Dmitrievič, Davydova, Natal'ja Naumovna, Ludikova, Anna Valer'evna, "Rekonstrukcija paleobiogeografičeskikh i paleoekologičeskikh uslovij oz. Džarylgač (Severo-Zapadnyj Krym) v pozdnem golocene po paleontologičeskim dannym " [«Reconstitution des conditions paléobiogéographiques et paléoécologiques du lac Džarylgač (Crimée du nord-ouest) durant l'Holocène tardif à la lumière des données paléontologiques"], in Paleontologija, paleobiogeografija i paleoekologija. Materialy LIII sessii Paleontologičeskogo Obščestva [Paléontologie, paléobiogéographie et paléoécologie. Matériaux de la LIII e session de la Société de Paléontologie], Sankt-Peterburg, Izdatel'stvo SPbGU, 2007, p. 89-90.

Nikolova, Alla Vladimirovna, PAškevič, Galina Aleksandrovna, «K voprosu ob urovne razvitija zemledelija Tripol'skoj kul'tury» ["A propos du niveau de développement de l'agriculture de la culture de Trypillja »], in Trypil'ski poselenn'ja-giganty. Materialy mižnarodnoj konferencii [Les établissements géants de Tripol'e. Matériaux de la conférence internationale], Kyïv, Korvin press, 2003, p. 89-95.

Osborne, Robin, Classical Landscape with Figures: The Ancient Greek City and its Countryside, London, George Philip, 1987.

PAšKevič, Galina Aleksandrovna, Paleoetnobotaničeskie nakhodki na territorii Ukrainy. Pamjatniki I tys. do n.è. - II tys. n.è. Katalog [Trouvailles paléobotaniques sur le territoire de l'Ukraine. Monuments du I ${ }^{e r}$ millénaire avant notre ère - II millénaire de notre ère. Un catalogue], Kiev, AN Ukrainy, 1991.

—, "Early Farming in the Ukraine», in Landscapes in Flux. Central and Eastern Europe in Antiquity, ed. by John Chapman, Pavel Dolukhanov, Oxford, Oxbow, 1997, p. 263-274. 
—, «New evidence for plant exploitation by the Scythian tribes during the Early Iron Age in the Ukraine", in Proceedings of the Fifth European Palaeobotanical and Palynological Conference: June 26-30, 1998, Krakow, ed. by Leon Stuchlik, Slawomir Florjan, Krakow, W. Szafer Institute of Botany, 1999, p. 597-601 (Acta Palaeobotanica. Suppl., 2).

-, "Archaeobotanical studies on the northern coast of the Black Sea", Eurasia Antiqua, 7 (2001), p. 511-567.

—, "Paleoetnobotanični doslidžennja Skhidnoj Ukraïny» [ Recherches paléobotaniques en Ukraine orientale»], in Problemy doslidžennja pam'jatok arkheologii Skhidnoj Ukraïny [Problèmes de la recherche des monuments archéologiques d'Ukraine orientale], Lugans'k, SNU im. V. Dalja, 2005, p. 11-14.

Podgorodeckij, Pjotr Dmitrievič, Severo-Zapadnyj Krym [La Crimée du nord-ouest], Simferopol', Tavrija, 1979.

Rammato, Dessalegn, Famine and Survival Strategies. A Case Study from Northeast Ethiopia, Uppsala, Nordiska Afrikainstitutet, 1991.

Rogov, Evgenij Jakovlevič, "Syrcovye konstrukcii v pogrebal'nykh sooruženijakh nekropolja Panskoe I» ["Constructions en brique crue employées dans les monuments funéraires de la nécropole de Panskoe I»], Kratkie soobščenija instituta arkheologii, 182 (1985), p. $45-50$.

—, «Nabor stoljarno-plotnič ikh instrumentov iz raskopok zdanija U6 poselenija Panskoe I» " "Un ensemble d'instruments de menuiserie-charpenterie des fouilles du bâtiment U6 de l'établissement de Panskoe I»], Arkheologičeskie Vesti, 7 (2000), p. 154-158.

—, "Metal Objects», in Panskoye I, Vol. 1: The Monumental Building U6, ed. by Lise Hannestad, Vladimir F. Stolba, Alexander N. Ščeglov, Aarhus, University Press, 2002, p. 252-267.

SApelko, Tatiana Valentinovna, Subetto, Dmitrij Aleksandrovič, Stolba, Vladimir Fjodorovič, "Vlijanie rel'efa na razvitie rastitel'nogo pokrova Kryma v golocene» " L'influence du relief sur le développement de la couverture végétale de la Crimée durant l'Holocène»], in Otečestvennaja geomorfologija: Prošloe, nastojaščee, buduščee. Materialy XXX Plenuma Geomorfologičeskoj komissii RAN [Géomorphologie nationale: Passé, présent, futur. Matériaux du XXX plénum de la Commission de géomorphologie de l'Académie des Sciences de Russie], otv. red. Dmitrij Valentinovič Lopatin, 
Sankt-Peterburg, Fakultet geografii i geoekologii SPbGU, 2008, p. 330-331.

SAPRYKin, Sergej Jur'evič, Denežnoe obraščenie na khore Khersonesa Tavričeskogo v antičnuju èpokhu [Circulation monétaire dans la chôra de Chersonèse Taurique durant l'Antiquité], Moskva, Institut Vseobščej Istorii RAN, 2005.

ŠčEglov, Aleksander Nikolaevič, "Issledovanie sel'skoj okrugi Kalos Limena» [Recherche du district agraire de Kalos Limèn»], Sovetskaja arkheologija, 3 (1967), p. 234-256.

—, «Zemel'nyj nadel u mysa Ojrat» ["Parcelles agricoles près du cap

Ojrat»], in Istorija i kul'tura antičnogo mira [Histoire et culture du monde antique], otv. red. Marija Michajlovna Kobylina, Moskva, Nauka, 1977, p. 210-215.

—, Severo-Zapadnyj Krym v antičnuju èpokhu [La Crimée du nord-ouest durant l'Antiquité], Leningrad, Nauka, 1978.

—, "Utilisation de la photographie aérienne dans l'étude du cadastre de Chersonésos Taurique (IVe-II s. av. n.è.)", Dialogues d'Histoire Ancienne, 6 (1980), p. 59-72.

—, "25 let rabot Tarkhankutskoj èkspedicii : itogi i perspektivy" ["25 années de travaux de la mission de Tarkhankut: résultats et perspectives»], Kratkie soob̌̌čenija instituta arkheologii, 182 (1985), p. 3-7.

—, «Un établissement rural en Crimée: Panskoje I (fouilles de 19691985)", Dialogues d'Histoire Ancienne, 13 (1987), p. 239-273.

—, «Issledovanija Tarkhankutskoj èkspedicii» [«Recherches de la mission de Tarkhankut»], in Arkheologičeskie issledovanija v Krymu: 1994 god [Recherches archéologiques en Crimée: 1994], Simferopol', 1997, p. 275-276.

—, "Cult sculpture, Altars, Sacred Vessels and Votivs", in Panskoye I, Vol. 1: The Monumental Building U6, ed. by Lise Hannestad, Vladimir F. Stolba, Alexander N. Ščeglov, Aarhus, University Press, 2002, p. 213-227.

—, "Monumental Building U6", in Panskoye I, Vol. 1: The Monumental Building U6, ed. by Lise Hannestad, Vladimir F. Stolba, Alexander N. Ščeglov, Aarhus, University Press, 2002, p. 27-98.

Ščeglov, Aleksander Nikolaevič, Rogov, Evgenij Jakovlevič, «Pogrebenija v podbojnykh mogilakh v Nižnem Pobuže, Nižnem Podnestrov’e i v Severo-Zapadnom Krymu» [Inhumations dans 
des tombes à niche dans le bas Boug, le bas Dniestr et en Crimée du nord-ouest»], in Problemy issledovanija Ol'vii [Problèmes de la recherche à Olbia], Parutino, 1985, p. 86-88.

Ščeglov, Aleksander Nikolaevič, Kuz'Minova, Natal'ja Stanislavovna, Januševič, Zoja Vasil'evna, ČavČavadze, Evgenia Savel'evna, "Zemledelie na poselenii Panskoe I (Severo-Zapadnyj Krym) v IV - načale III v. do n.è.» [ L'agriculture dans l'établissement de Panskoe I (Crimée du nord-ouest) au IV $\mathrm{V}^{\mathrm{e}}$ - début du III ${ }^{\mathrm{e}}$ siècle avant notre ère»], in Flora i rastitel'nost' [Flore et végétation], Kišinev, Štiinca, 1989, p. 50-69 (Botaničeskie issledovanija, 5).

Ščeglov, Aleksander Nikolaevič, Hannestad, Lise, Stolba, Vladimir Fjodorovič, "Raboty rossijsko-datskoj Tarkhankutskoj èkspedicii v Krymu» ["Travaux de la mission russo-danoise de Tarkhankut en Crimée»], in Arkheologičeskie otkrytija 1994 g. [Découvertes archéologiques de 1994], Moskva, 1995, p. 335-337.

—, «Raskopki poselenija Panskoe I v Krymu» ["Fouilles de l'établissement de Panskoe I en Crimée»], in Izučenie kul'turnykh vzaimodejstvij i novye archeologičeskie otkrytija [Etude des interactions culturelles et nouvelles découvertes archéologiques], otv. red. Vadim Mikhajlovič Masson, Sankt-Peterburg, 1995, p. 50-53.

Ščeglov, Aleksander Nikolaevič, Hannestad, Lise, Kašaev, Sergej Vladimirovič, Kirco, Ljubov' Borisovna, Sørensen, Jacob, Stolba, Vladimir Fjodorovič, Hastrup, Helene, «Rossijskodatskie raskopki na poselenii i nekropole Panskoe I» ["Fouilles russo-danoises de l'établissement et de la nécropole de Panskoe I»], Arkheologičeskie Vesti, 4 (1995), p. 288-290.

Smekalova, Tatiana Nikolaevna, Stolba, Vladimir Fjodorovič, Materials for the Archaeological Map of Crimea. I. Monuments of the Bronze Age and Early Iron Age on the Tarkhankut Peninsula: Preliminary Report, Simferopol', Dolja, 2009.

Sмith, Philip M., "Minor Crops", in Evolution of Crop Plants, ed. Norman W. Simmonds, London/New York, Longman, 1976, p. 301-324.

SNODGRASs, Anthony, «Survey Archaeology and the Rural Landscape of the Greek City", in The Greek City from Homer to Alexander, ed. by Oswyn Murray, Simon Price, Oxford, Clarendon Press, 1990, p. 113-136. 
Stolba, Vladimir Fjodorovič, "Novoe posvjaščenie iz SeveroZapadnogo Kryma i aspekty kul'ta Gerakla v Khersonesskom gosudarstve» ["Nouvelle dédicace de la Crimée du nord-ouest et aspects du culte d'Héraclès dans l'Etat de Chersonèse »], Vestnik Drevnej Istorii, 4 (1989), p. 55-70.

—, «Dom IV v. do n.è. na poselenii Panskoe I : raskopki 1987 g.» ["Une maison du IV ${ }^{\mathrm{e}}$ siècle avant notre ère sur l'établissement de Panskoe I: fouilles de 1987 », Kratkie soobščenija instituta arkheologii, 204 (1991), p. 78-84.

—, "Graffiti and Dipinti», in Panskoye I, Vol. 1: The Monumental Building U6, ed. by Lise Hannestad, Vladimir F. Stolba, Alexander N. Ščeglov, Aarhus, University Press, 2002, p. 228-244.

—, "Handmade Pottery", in Panskoye I, Vol. 1: The Monumental Building U6, ed. by Lise Hannestad, Vladimir F. Stolba, Alexander N. Ščeglov, Aarhus, University Press, 2002, p. 180-200.

—, "Grečeskoe pis'mo s poselenija Panskoe I (Severo-Zapadnyj Krym)» ["Une lettre grecque de l'établissement de Panskoe I (Crimée du nord-ouest)»], Vestnik Drevnej Istorii, 4 (2005), p. 76-87.

-, "Monetary Crises in the Early Hellenistic Poleis of Olbia, Chersonesos and Pantikapaion. A Re-assessment", in XIII Congreso Internacional de Numismática (Madrid, 2003). Actas, ed. por Carmen Alfaro, Carmen Marcos, Paloma Otero, Madrid, Ministerio de Cultura, 2005, p. 395-403.

—, "The Oath of Chersonesos and the Chersonesean Economy in the Early Hellenistic Period", in Making, Moving and Managing. The New World of Ancient Economies, 323-31 BC, ed. by Zofia G. Archibald, John K. Davies, Vincent Gabrielsen, Oxford, Oxbow, 2005, p. 298-321.

—, «Kauri i pročie amulety v pogrebal'nom obrjade nekropolja Panskoe I" [Cypraeides et autres amulettes dans le rituel funéraire de la nécropole de Panskoe I»], in Bosporskij fenomen. Sakral'nyj smysl regiona, pamjatnikov, nakhodok. Materialy meždunarodnoj naučnoj konferencii [Le phénomène bosporan. Signification sacrée de la région, des monuments, des trouvailles. Actes $d u$ colloque scientifique international], otv. red. Vadim Jur'evič Zuev et al., Sankt-Peterburg, vol. 2, p. 157-162 et 382-383. 
—, «Busy, podveski i amulety: Vera v sglaz u grečeskogo i mestnogo naselenija Tavriki» " Colliers, pendentifs et amulettes : croyance en le mauvais œil auprès de la population grecque et indigène de Tauride»], Vestnik drevnej istorii, 2 (2009), p. 109-128.

—, «Multicultural Encounters in the Greek Countryside: Evidence from the Panskoye-I Necropolis, Western Crimea", in Pontika 2008. Recent Research on the Northern and Eastern Black Sea in Ancient Times, ed. by Michael Vickers, Ewdoksia Papuci-Władyka, Jarosław Bodzek, David Braund, Oxford, Archaeopress, 2011, p. 329-340 (BAR Int. Ser. 2240).

Stolba, Vladimir Fjodorovič, Golencov, Anatolij Sergeevič, «Monety iz raskopok i slučajnykh nakhodok na Južno-Donuzlavskom gorodišče (Severo-Zapadnyj Krym)»["Monnaies provenant de fouilles et de trouvailles fortuites sur le site de JužnoDonuzlavskoe (Crimée du nord-ouest)»], in $\Sigma \Upsilon \Sigma \Sigma$ ITIA. Pamjati Jurija Viktoroviča Andreeva [ $\Sigma \Upsilon \Sigma \Sigma$ ITIA. En mémoire de Jurij Viktorovič Andreev], otv. red. Vadim Jur'evič Zuev, SanktPeterburg, Aletejja, 2005, p. 274-280.

Stolba, Vladimir F., Rogov, Eugeny, Panskoye I, Vol. 2: The Necropolis, Aarhus, University Press, 2012.

StrŽeleckij, Stanislav Francevič, Klery Khersonesa Tavričeskogo. K istorii drevnego zemledelija v Krymu [Les lots de terre de Chersonèse Taurique. De l'histoire de l'agriculture antique en Crimée], Simferopol', Krymizdat, 1961.

Subetto, Dmitrij Alexandrovič, Sapelko, Tatiana Valentinovna, Stolba, Vladimir Fjodorovič, «Issledovanija paleolimnologov v Krymu» [ Recherches des paléolimnologues en Crimée»], Priroda, 12 (2007), p. 61-62.

Subetto, Dmitrij A., Stolba, Vladimir F., Neustrueva, Irina Yu., Sapelko, Tatiana V., Kuznecov, Denis D., Gerasimenko, Natalia P., Baкhmutov, Vladimir G., Ludikova, Anna V., Davydova, Natalia N., «Environmental and Black Sea Level Changes in the Holocene as recorded in Lakes Saki and Dzharylgach, Crimean Peninsula", in Extended abstracts IGCP 521-481 Joint Meeting and Field Trip, Gelendzhik-Kerch, September 8-17, 2007, Moscow, 2007, p. 157-159.

Subetto, Dmitrij Aleksandrovič, Gerasimenko, Natal'ja Petrovna, Bakhmutov, Vladimir Georgievič, Neustrueva, Irina 
Jur'evna, Sapelko, Tatiana Valentinovna, Kuznecov, Denis Dmitrievič, Davydova, Natalja Naumovna, Ludikova, Anna Valer'evna, Stolba, Vladimir Fjodorovič, "Rekonstrukcija paleogeografičnykh umov Zakhidnogo Krymu u pizn'omu goloceni za litologičnymy i paleontologičnymy materialamy vyvčennja ozer» "Reconstitution des conditions paléogéographiques de la Crimée occidentale durant l'Holocène tardif à la lumière des matériaux lithologiques et paléontologiques de l'étude des lacs »], Fizyčna geografija ta geomorfologija, 56 (2009), p. 299-310.

Vysotskaja, Tatiana Nikolaevna, «Drevnegrečeskoe poselenie v pos.

Mežvodnoe» " Un établissement grec antique dans le village de Mežvodnoe»], in Arkheologičeskie otkrytija 1968 g. [Découvertes archéologiques en 1968], Moskva, 1969, p. 295-296.

Wasylikowa, Krystyna, Carciumaru, Marin, Hajnalova, Eva, Hartyanyi, Borbala P., Pashrevich, Galina A., Yanushevich, Zoya V., "East-Central Europe», in Progress in Old World Palaeoethnobotany, ed. by Willem van Zeist, Krystyna Wasylikowa, Karl-Ernst Behre, Rotterdam, Balkema, 1991, p. 207-239.

Wood, Joseph S., Knight, C. Gregory, "Cross cultural perspectives on human adjustments to arid margins", in Drought, Famine and Population Movements in Africa, ed. by James L. Newman, Syracuse N.Y., Syracuse University, 1975, p. 75-126.

ZeEst, Iraida Borisovna, "Arkhaičeskie sloi Germonassy» ["Les couches archaïques de Hermonassa»], Kratkie soob̌̌čenija instituta arkheologii, 83 (1965), p. 53-58.

Žuravl’jov, Oleg Petrovič, Istorija fauny i tvarynnyctva Nyžn'ogo Pobužž ja v antyčnyj čas. Avtoref. kand. diss. [Histoire de la faune et élevage du bas Boug durant l'Antiquité. Résumé de dissertation], Kyïv, 1993.

\section{Crédits iconographiques}

Sauf mention contraire, les figures sont propriétés de l'auteur. 